Supplementary Information for:

\title{
A General Decomposition Pathway for Phosphine-Stabilized Metathesis Catalysts: Lewis Donors Accelerate Methylidene Abstraction
}

\author{
William L. McClennan, Stephanie A. Rufh, Justin A. M. Lummiss, and Deryn E. Fogg*
}

Department of Chemistry and Centre for Catalysis Research \& Innovation, University of Ottawa, Ottawa, Ontario, Canada K1N 6N5

*Corresponding author. Email: dfogg@uottawa.ca

\section{Table of Contents}

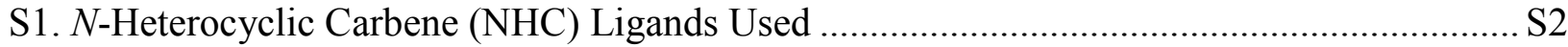

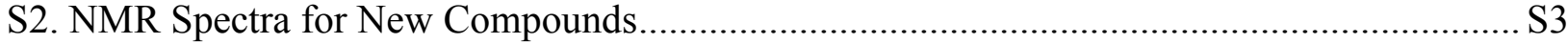

S3. Representative NMR Spectra for Transient $\sigma$-Alkyl Species............................................ 7

S4. Donor-Accelerated Methylidene Abstraction: Scope with Respect to Catalyst. Representative

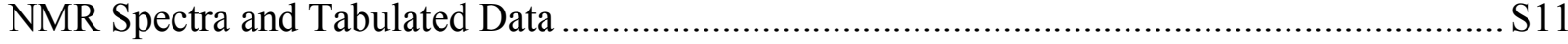

S5. Donor-Accelerated Methylidene Abstraction: Scope with Respect to Donor. Representative

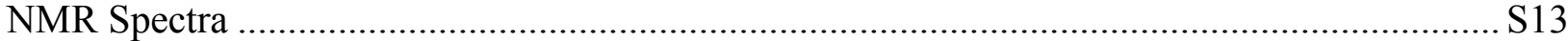

S6. Kinetics Data for Decomposition of Methylidene Complexes ........................................ S15

S7. NMR Spectra Relating to Points Discussed in Text....................................................... S18

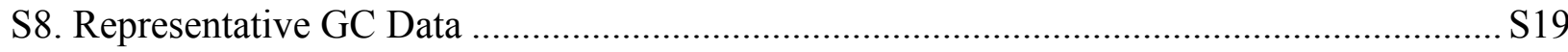

S9. NMR Spectra for Experiments with Ethylidene Complexes ....................................... S20

S10. Formation of [ $\left.\mathrm{MePCy}_{3}\right] \mathrm{Cl} \mathbf{A}$ by Water-Promoted Decomposition during Catalysis ......... S22

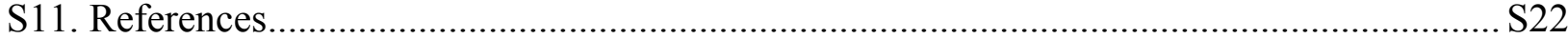


S1. N-Heterocyclic Carbene (NHC) Ligands Used
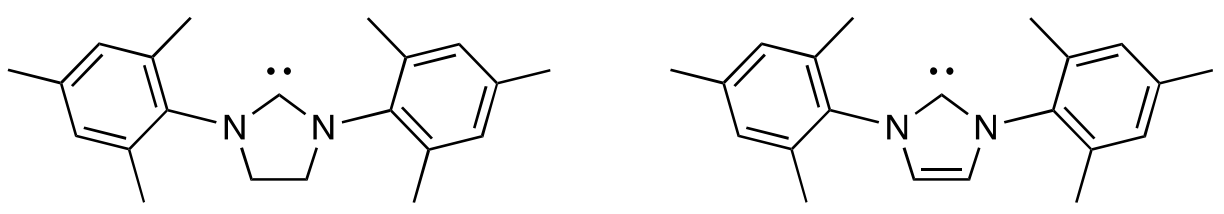

$\mathrm{H}_{2}$ IMes

IMes
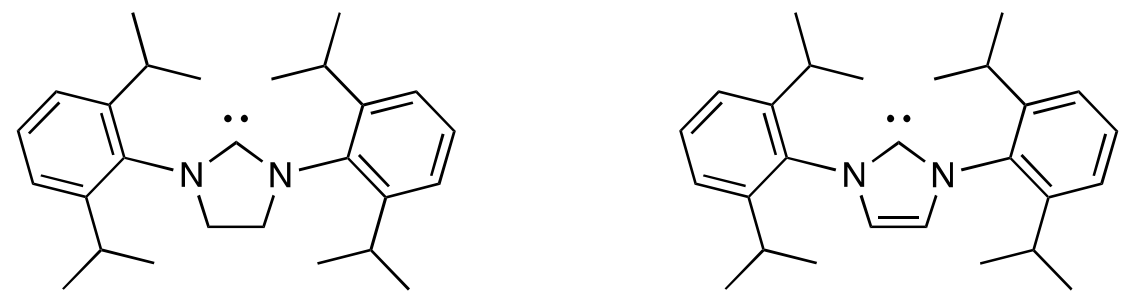

$\mathrm{H}_{2} \mathrm{IPr}$

IPr

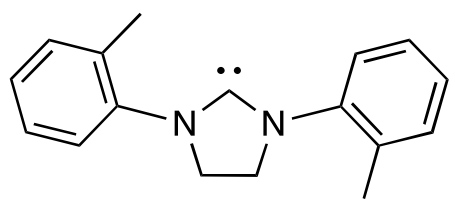

$\mathrm{H}_{2}$ ITol<smiles>[2H]c1c([2H])c(C([2H])([2H])[2H])c(N2CCN(c3c([2H])c([2H])c(C([2H])([2H])[2H])c([2H])c3C([2H])([2H])[2H])C2)c(C([2H])([2H])[2H])c1[2H]</smiles>

$d_{22}-\mathrm{H}_{2}$ IMes

Figure S1. Structures and codes for NHC ligands described in the text. 


\section{S2. NMR Spectra for New Compounds}

(a) ${ }^{1} \mathrm{H}$ NMR spectrum $\left(300 \mathrm{MHz}, \mathrm{C}_{6} \mathrm{D}_{6}\right)$.

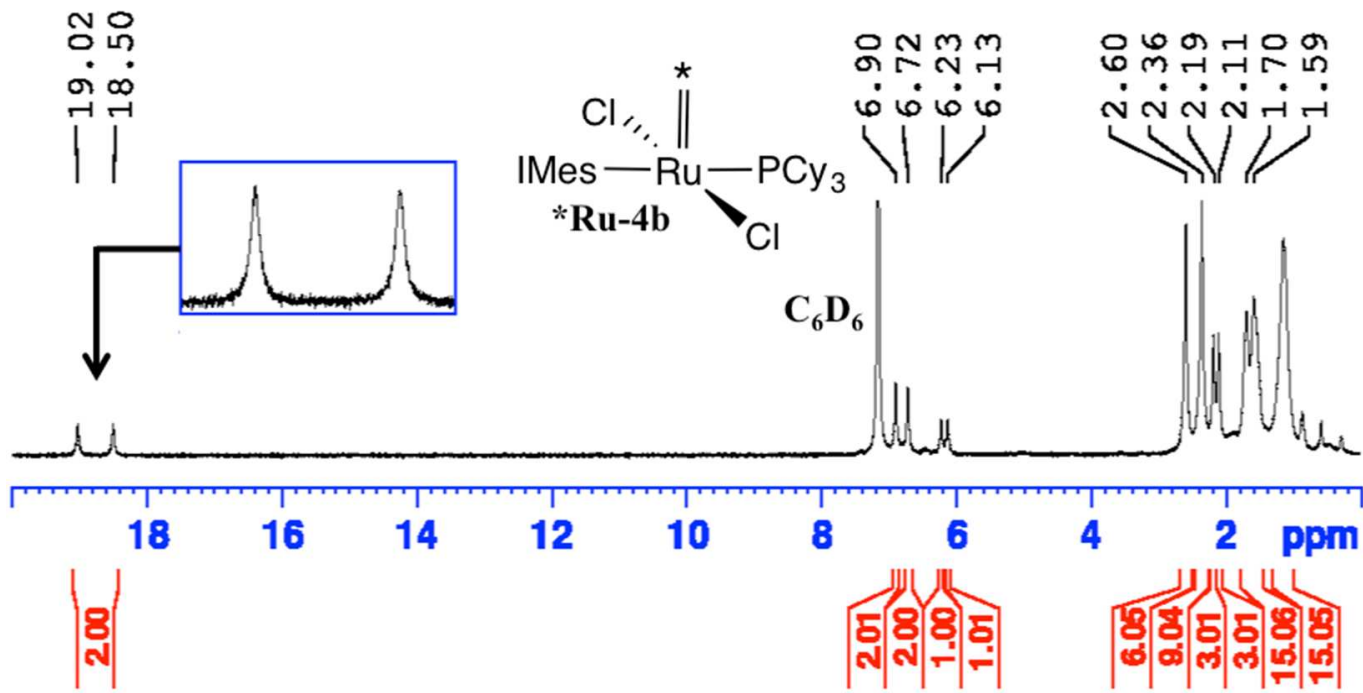

(b) ${ }^{13} \mathrm{C}\left\{{ }^{1} \mathrm{H}\right\}$ NMR spectrum $\left(75 \mathrm{MHz}, \mathrm{C}_{6} \mathrm{D}_{6}\right)$, showing methylidene signal.

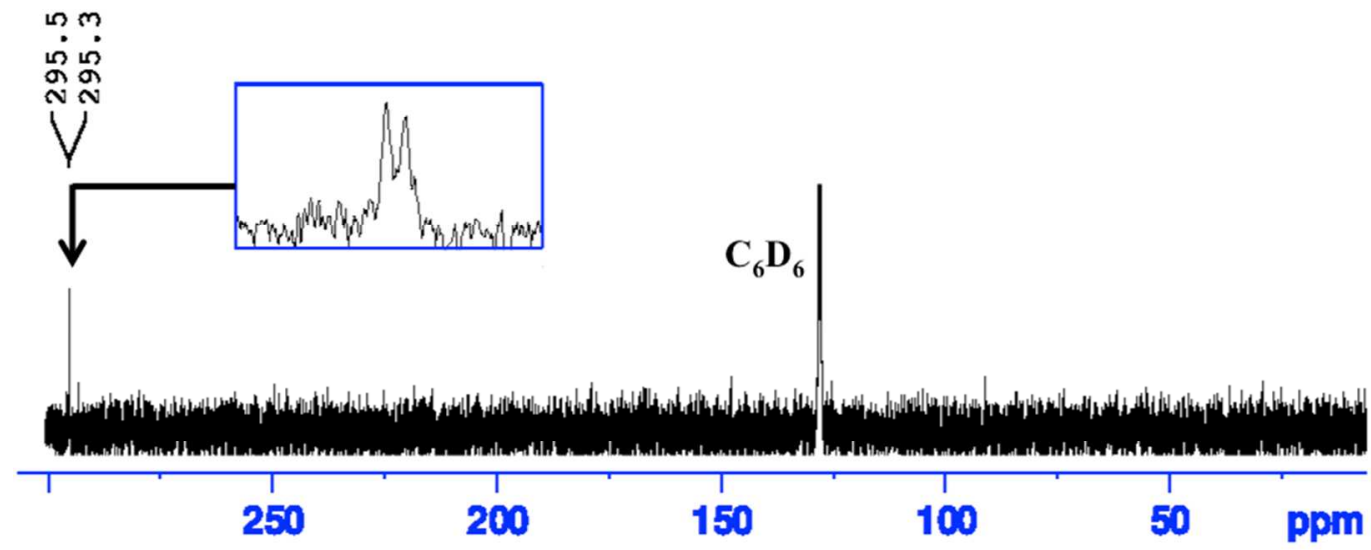

(c) ${ }^{31} \mathrm{P}\left\{{ }^{1} \mathrm{H}\right\}$ NMR spectrum $\left(121 \mathrm{MHz}, \mathrm{C}_{6} \mathrm{D}_{6}\right)$.

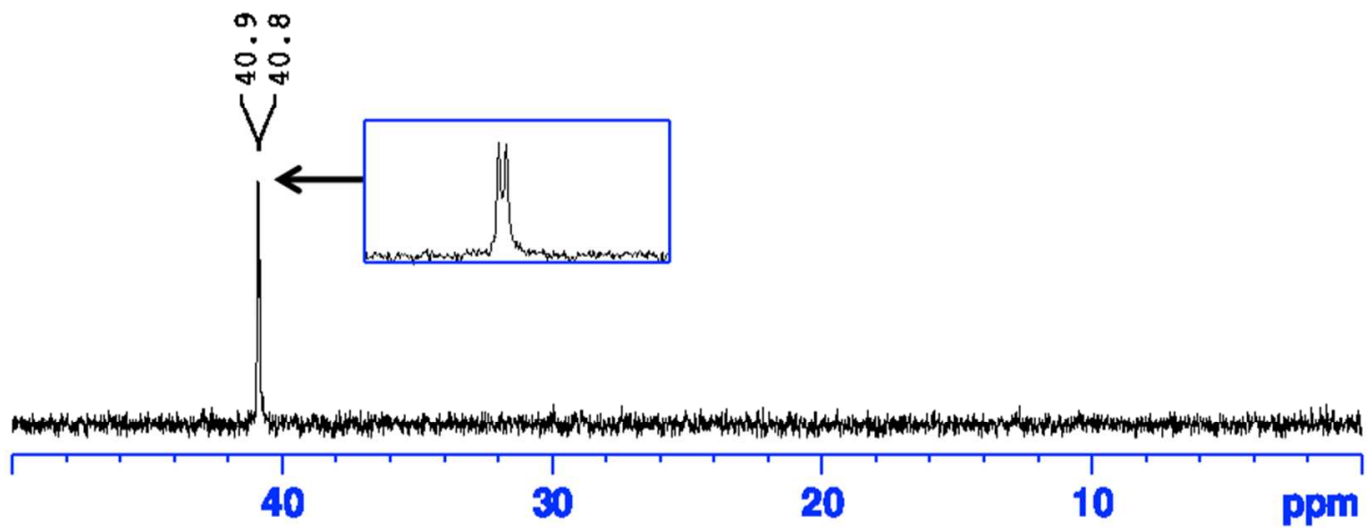

Figure S2. Characterization of $\mathrm{RuCl}_{2}(\mathrm{IMes})\left(\mathrm{PCy}_{3}\right)\left(={ }^{13} \mathrm{CH}_{2}\right),{ }^{*} \mathbf{R u}-\mathbf{4 b}$. 
(a) ${ }^{1} \mathrm{H}$ NMR (300 MHz, $\left.\mathrm{C}_{6} \mathrm{D}_{6}\right)$.

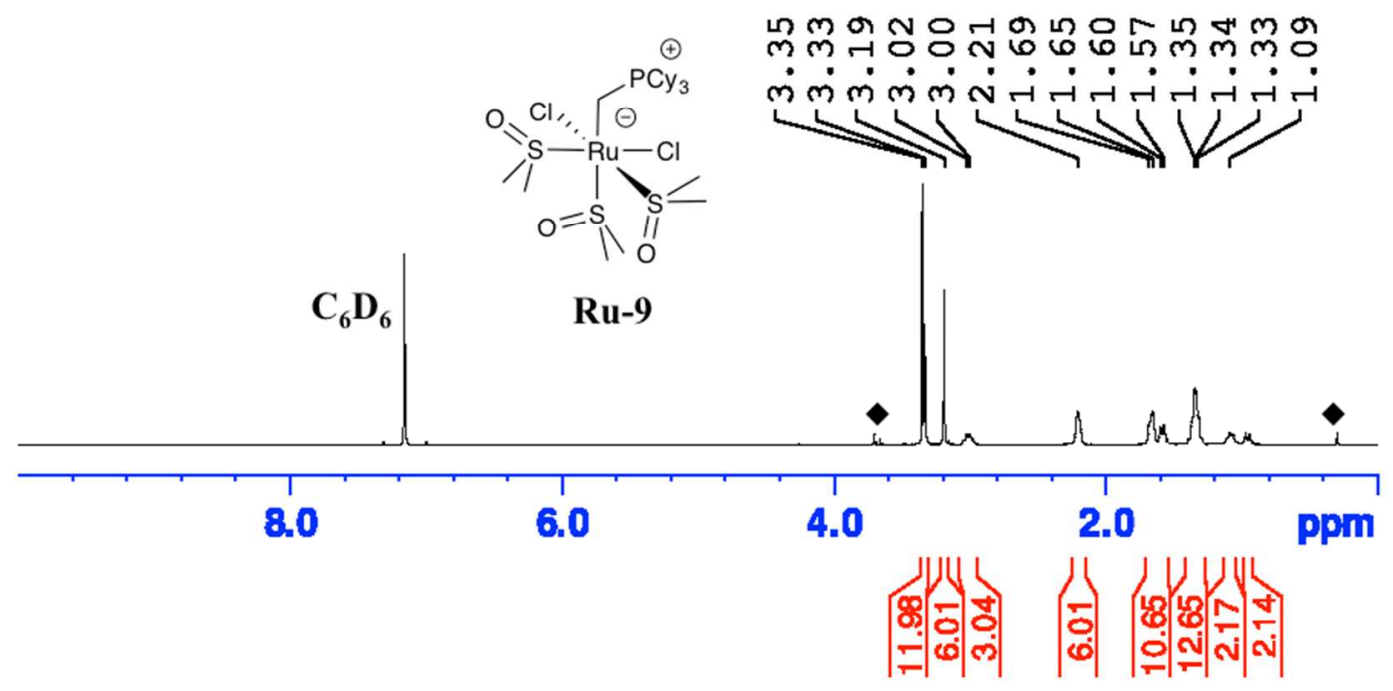

(b) ${ }^{13} \mathrm{C}\left\{{ }^{1} \mathrm{H}\right\}$ NMR spectrum (75 MHz, $\left.\mathrm{C}_{6} \mathrm{D}_{6}\right)$.

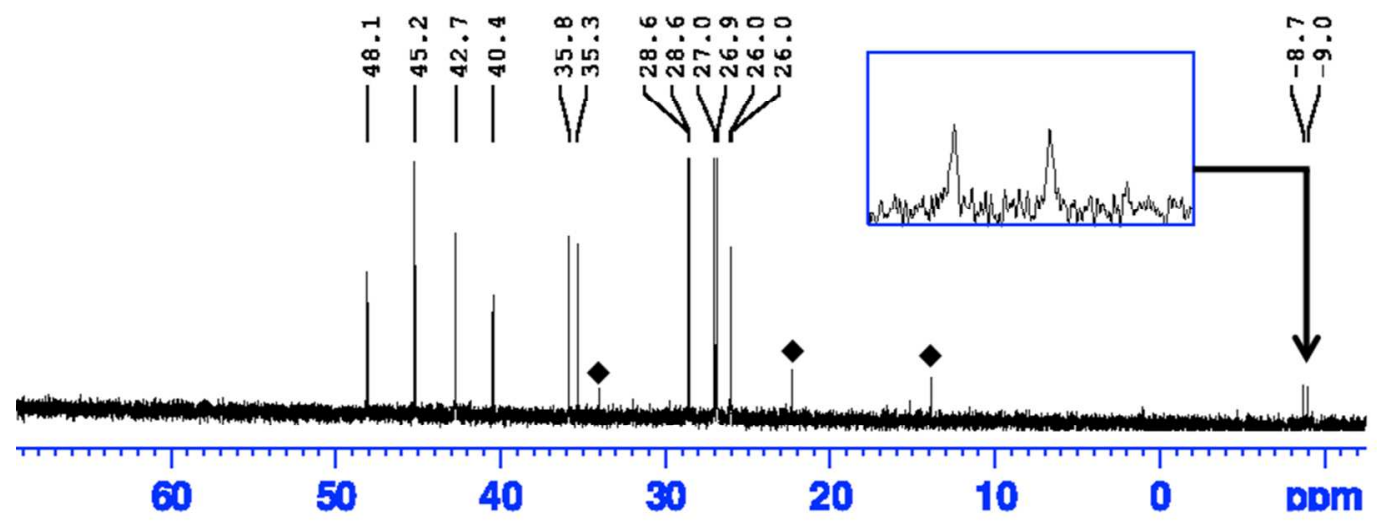

(c) ${ }^{31} \mathrm{P}\left\{{ }^{1} \mathrm{H}\right\}$ NMR spectrum $\left(121 \mathrm{MHz}, \mathrm{C}_{6} \mathrm{D}_{6}\right)$.

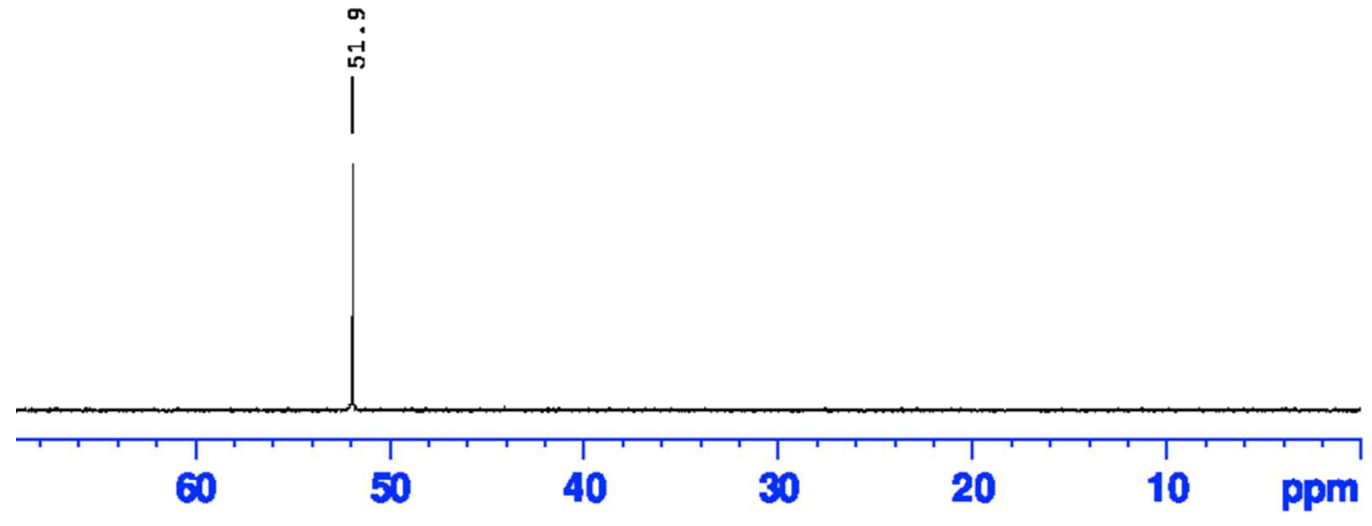

Figure S3. Characterization of $\mathrm{RuCl}_{2}\left(\sigma-\mathrm{CH}_{2} \mathrm{PCy}_{3}\right)(\mathrm{DMSO})_{3} \mathbf{R u}-\mathbf{9}$. Minor amounts of residual solvent, which could not be removed despite drying under vacuum for $12 \mathrm{~h}$, are denoted with $(\bullet)$. 


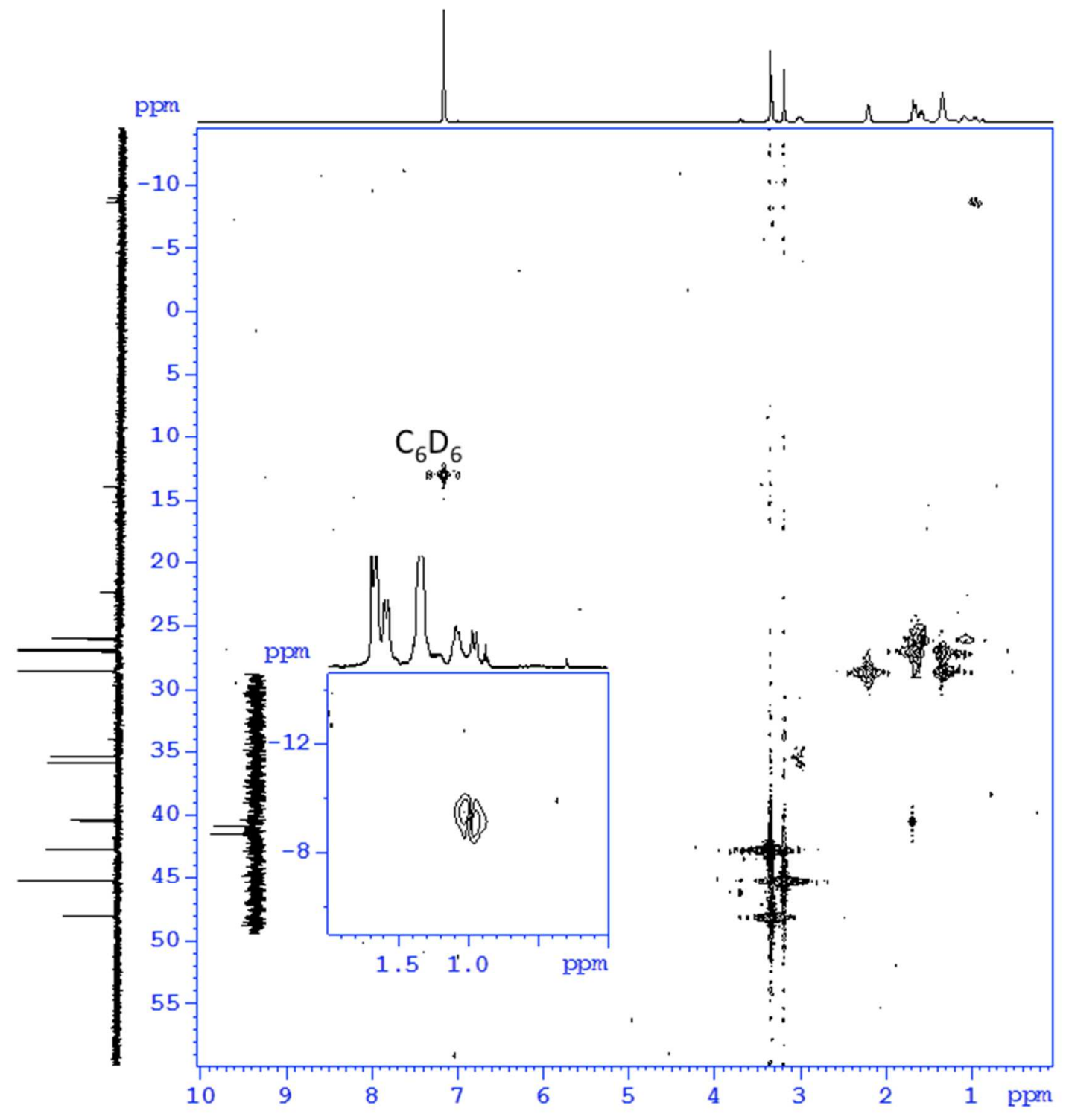

Figure S4. ${ }^{1} \mathrm{H}-{ }^{13} \mathrm{C}$ HMQC spectrum $\left(\mathrm{C}_{6} \mathrm{D}_{6}\right)$ for $\mathrm{RuCl}_{2}\left(\sigma-\mathrm{CH}_{2} \mathrm{PCy}_{3}\right)(\mathrm{DMSO})_{3} \mathbf{R u - 9}$. Inset shows ${ }^{1} J_{\mathrm{C}-\mathrm{H}}$ correlations within the $\mathrm{Ru}-\mathrm{CH}_{2}$ group. 


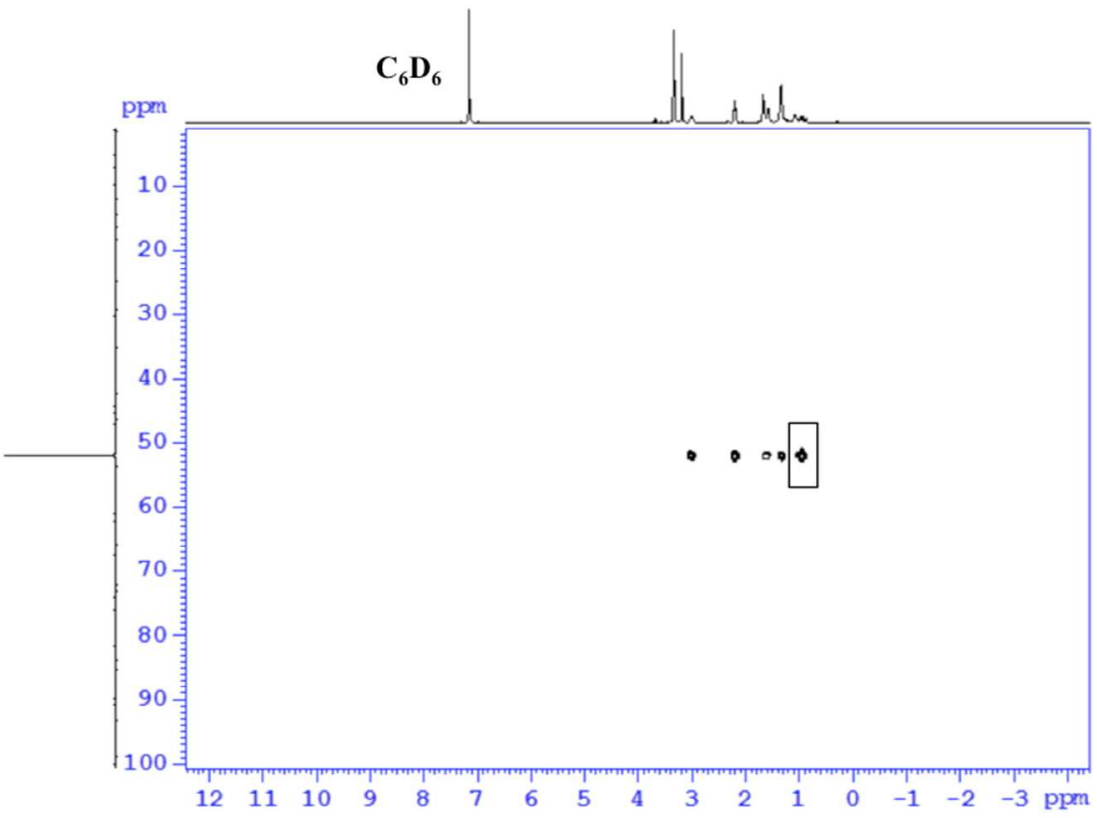

Figure S5. ${ }^{1} \mathrm{H}^{-31} \mathrm{P}$ HMQC $\left(\mathrm{C}_{6} \mathrm{D}_{6}\right)$ spectrum of $\left.\mathrm{RuCl}_{2}\left(\sigma-\mathrm{CH}_{2} \mathrm{PCy}\right)_{3}\right)(\mathrm{DMSO})_{3} \mathbf{R u}-\mathbf{9}$. Box shows the two-bond ${ }^{2} J_{\mathrm{H}-\mathrm{P}}$ correlation within the $\mathrm{Ru}-\mathrm{CH}_{2} \mathrm{PCy}_{3}$ group.

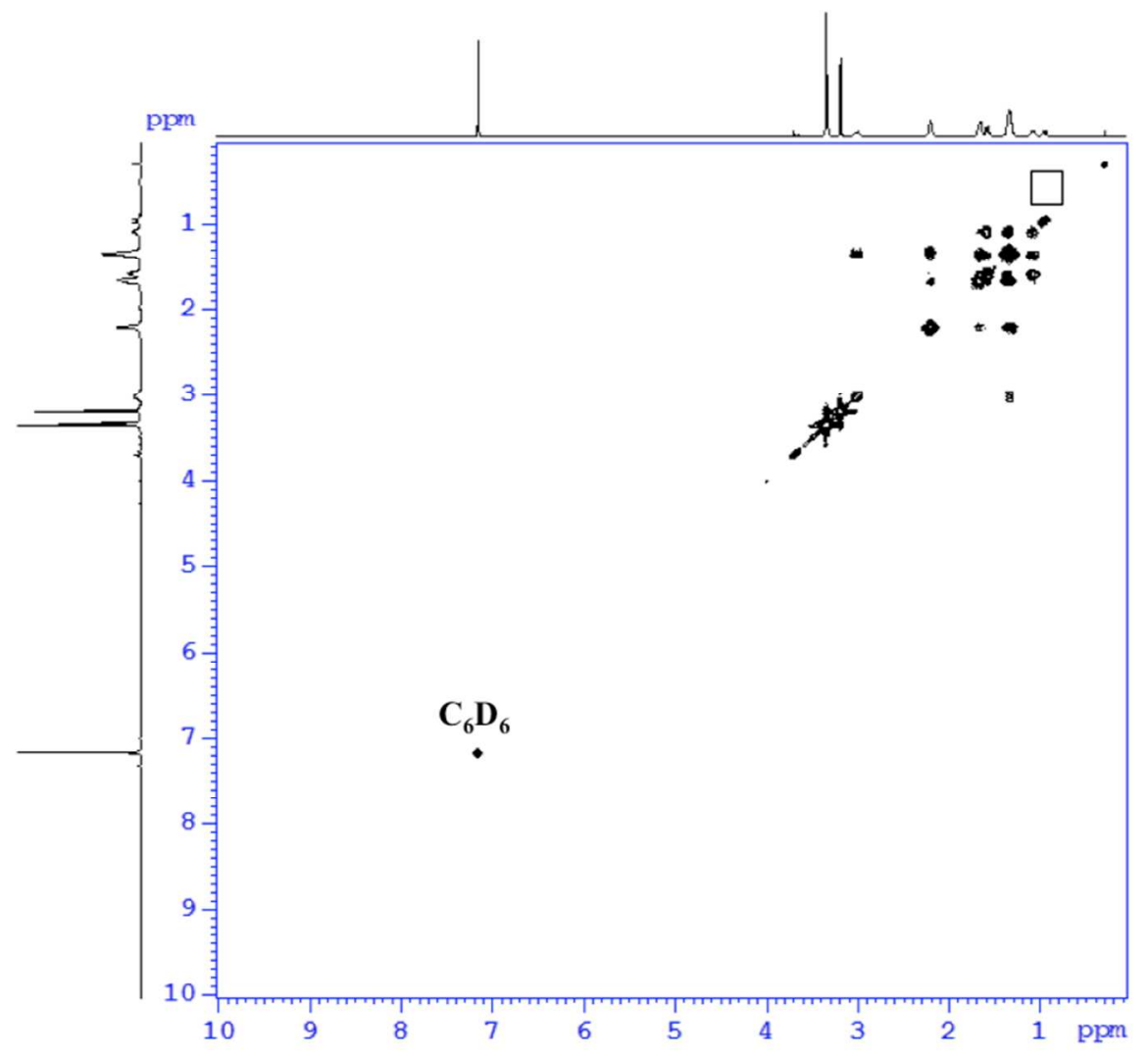

Figure S6. ${ }^{1} \mathrm{H}^{-1} \mathrm{H} \operatorname{COSY}\left(300 \mathrm{MHz}, \mathrm{C}_{6} \mathrm{D}_{6}\right)$ spectrum of $\mathrm{RuCl}_{2}\left(\sigma-\mathrm{CH}_{2} \mathrm{PCy}_{3}\right)(\mathrm{DMSO})_{3}$ Ru-9. Box shows the absence of any additional $\mathrm{H}-\mathrm{H}$ correlations for the methylene protons highlighted, consistent with assignment to $\mathrm{Ru}-\mathrm{CH}_{2} \mathrm{PCy}$. 
S3. Representative NMR Spectra for Transient $\sigma$-Alkyl Species
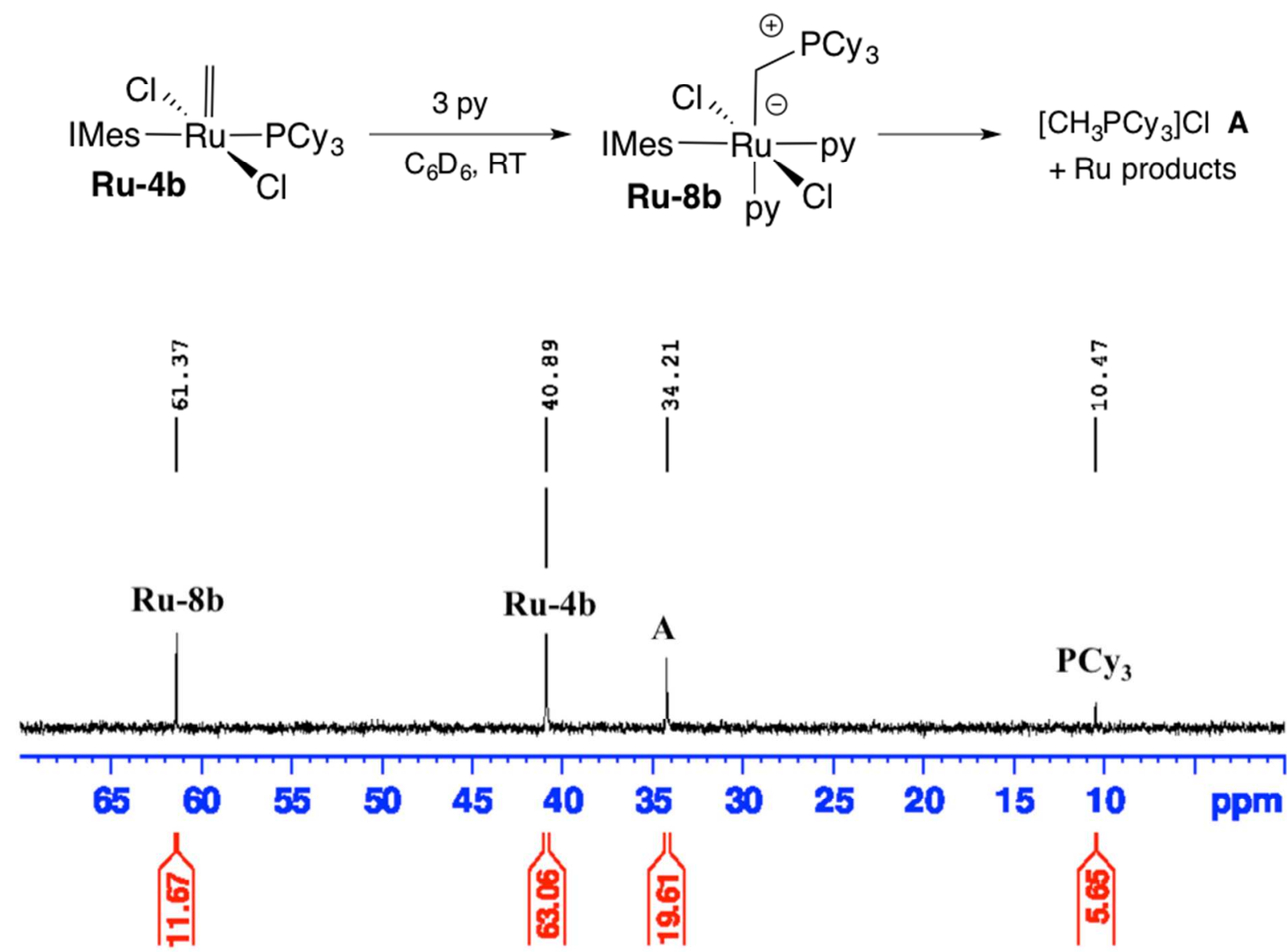

Figure S7. Reaction (top) and ${ }^{31} \mathrm{P}\left\{{ }^{1} \mathrm{H}\right\}$ NMR spectrum (121 MHz, $\left.\mathrm{C}_{6} \mathrm{D}_{6}\right)$ showing signal for transient $\mathbf{R u - 8 b}$, observed within 5 min of treating non-labelled $\mathbf{R u}-\mathbf{4 b}$ with 3 equiv pyridine.

Note: Spectrum corresponds to values shown in red in Table S2, p. S15. 


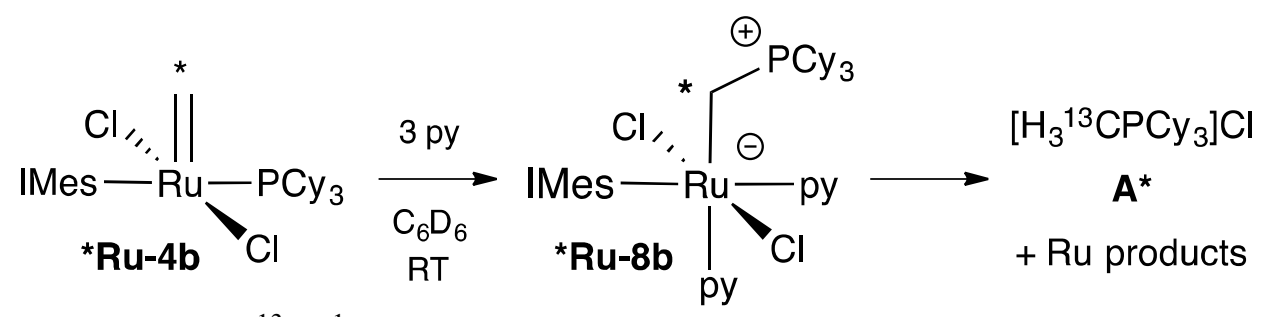

(a) 2-16 min (400 scans): ${ }^{13} \mathrm{C}\left\{{ }^{1} \mathrm{H}\right\}$ NMR spectrum (75 MHz, $\left.\mathrm{C}_{6} \mathrm{D}_{6}\right)$, key signals only.

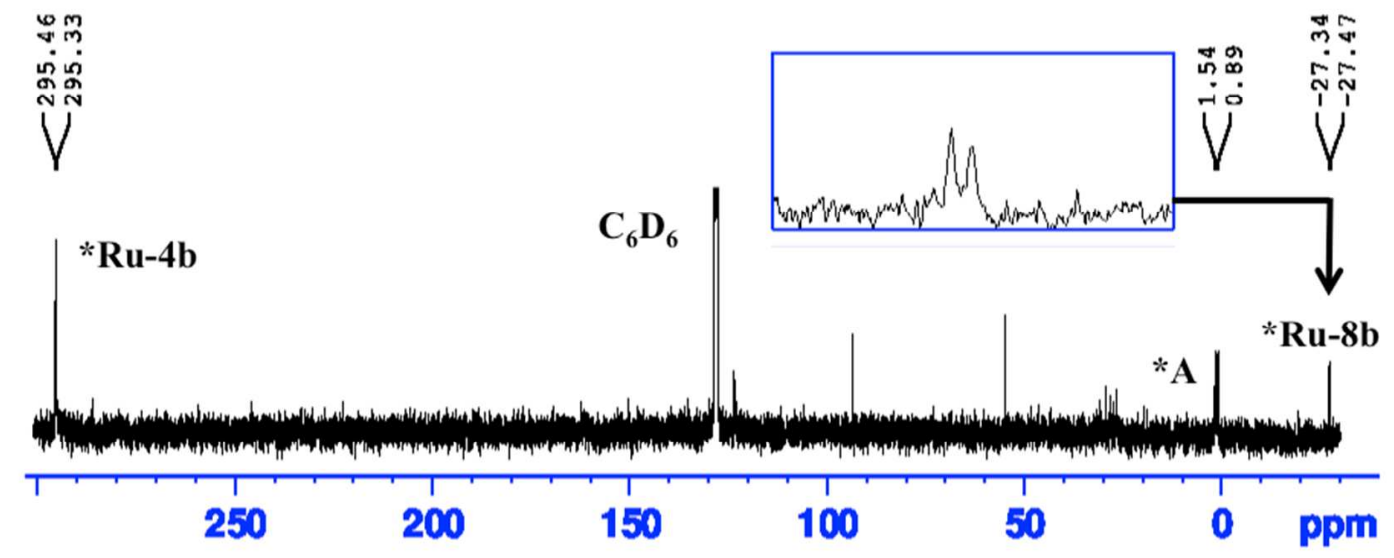

(b) 16-22 min: ${ }^{31} \mathrm{P}\left\{{ }^{1} \mathrm{H}\right\}$ NMR spectrum (121 MHz, $\left.\mathrm{C}_{6} \mathrm{D}_{6}\right)$.

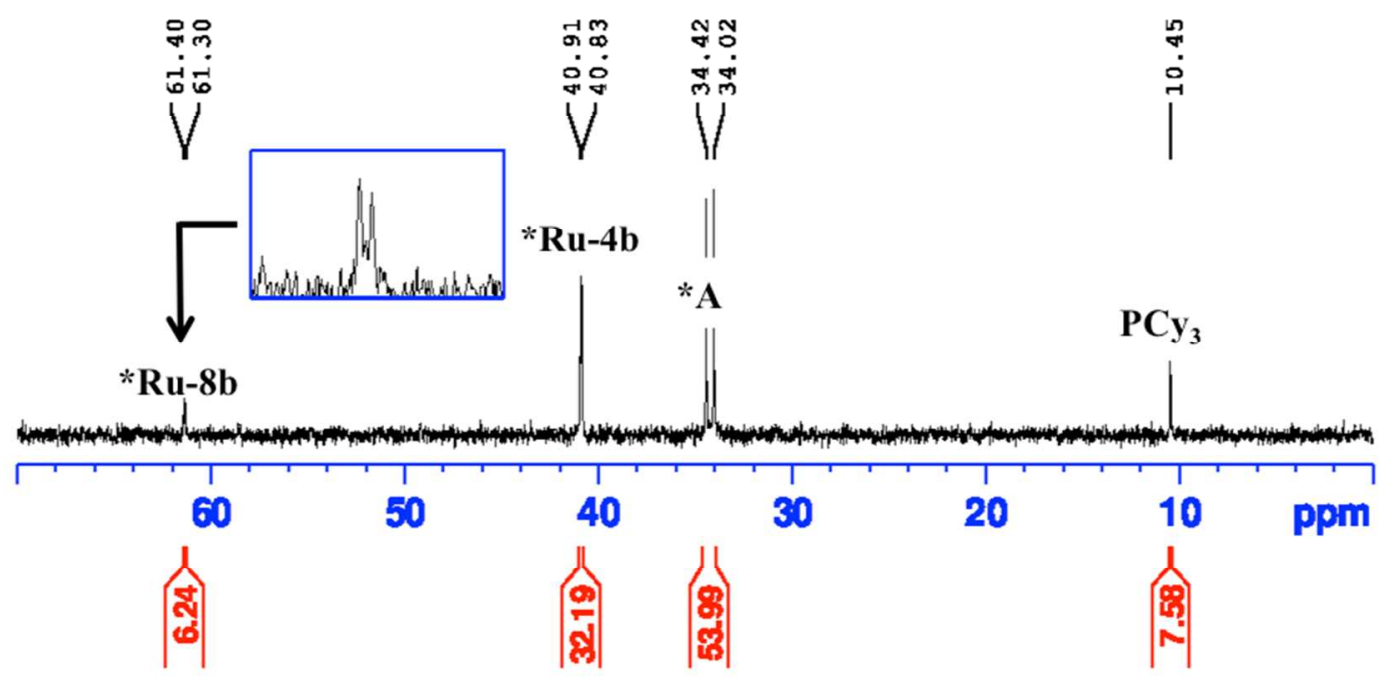

Figure S8. Reaction (top) and NMR spectra showing formation of transient $* \mathbf{R u}-\mathbf{8 b}$ on treating ${ }^{13}$ C-labelled * Ru-4b with 3 equiv pyridine.

Note: for spectra showing the decomposition products formed on treating $\mathrm{RuCl}_{2}\left(\mathrm{H}_{2} \mathrm{IMes}\right)\left(\mathrm{PCy}_{3}\right)\left(={ }^{13} \mathrm{CH}_{2}\right) * \mathbf{R u}-\mathbf{4 a}$ with pyridine, see the prior Communication. ${ }^{1}$ 


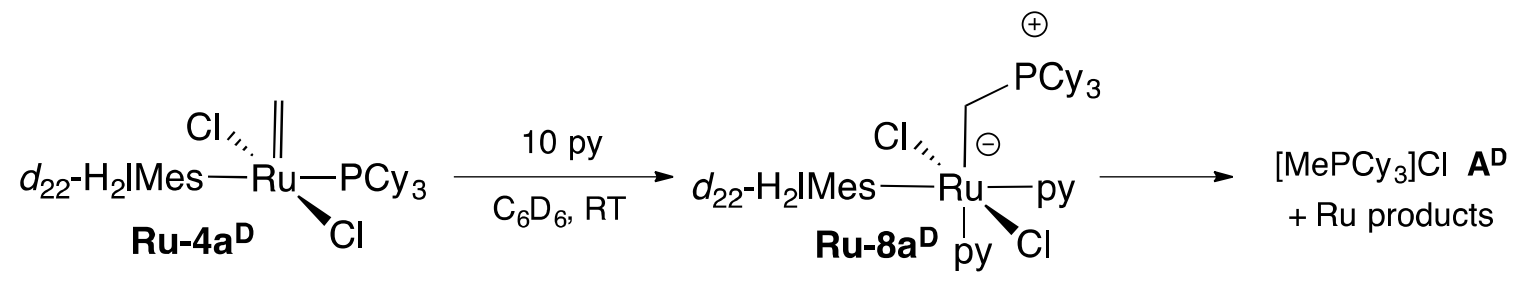

(a) Spectrum obtained within 5 min, showing signal for transient $\mathbf{R u} \mathbf{u}-\mathbf{a}^{\mathbf{D}}$.

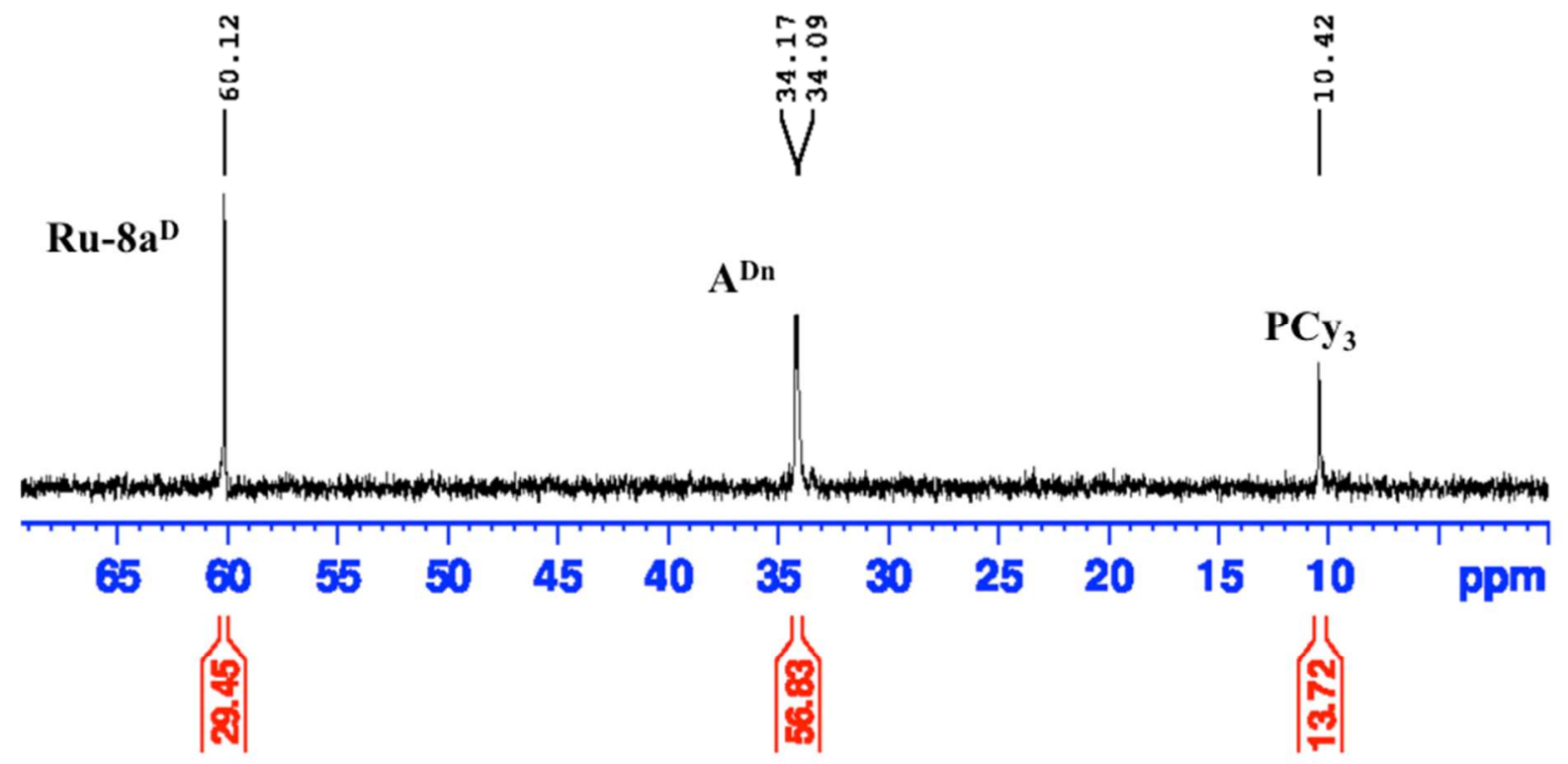

(b) Spectrum obtained after 10 min, showing full decomposition.

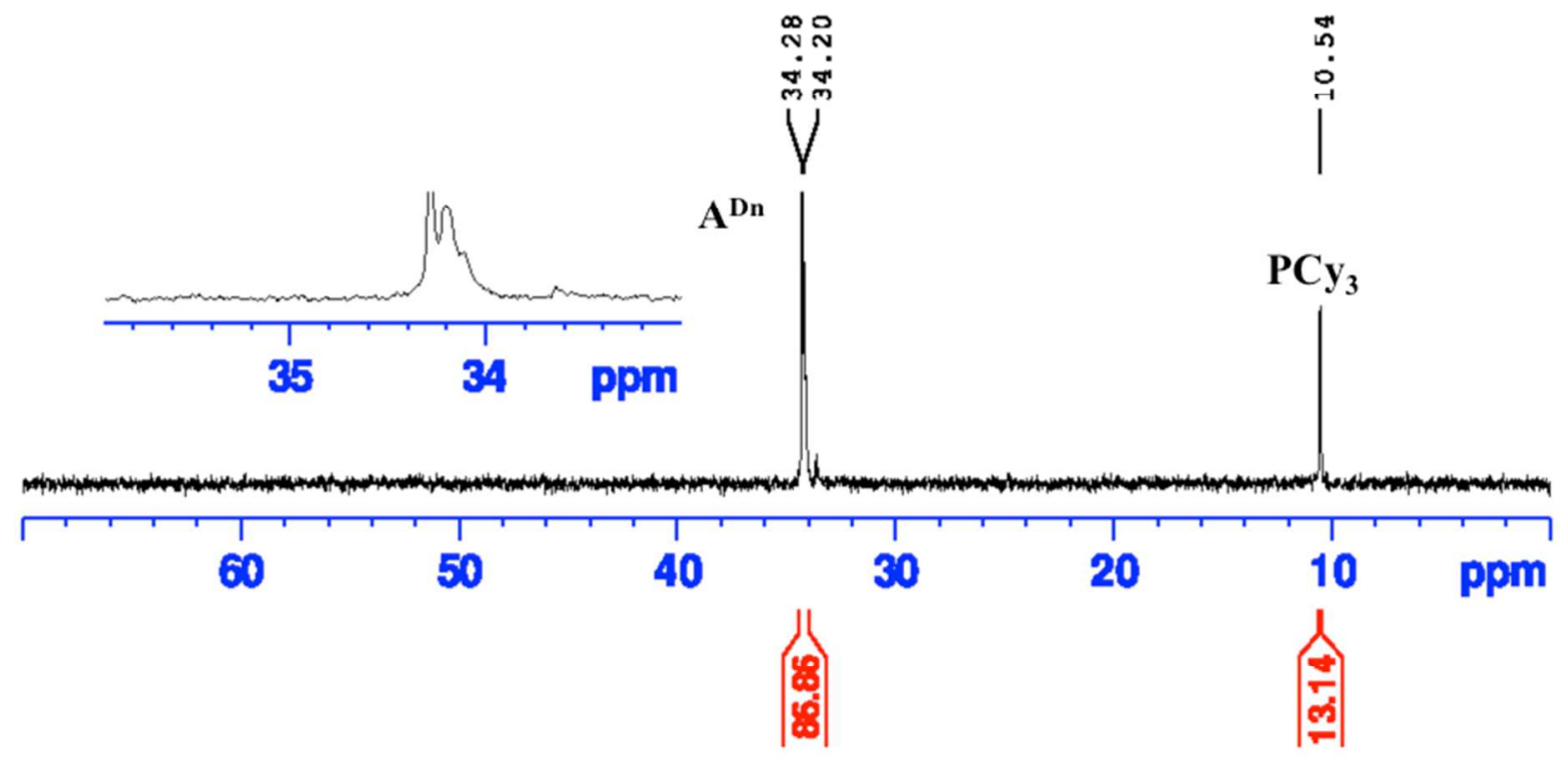

Figure S9. Reaction (top) and ${ }^{31} \mathrm{P}\left\{{ }^{1} \mathrm{H}\right\}$ NMR spectra (121 MHz, $\mathrm{C}_{6} \mathrm{D}_{6}$ ) obtained on treating deuterium-labelled $\mathbf{R u}-\mathbf{4} \mathbf{a}^{\mathbf{D}}$ with pyridine (10 equiv). The signal for phosphonium salt $\mathbf{A}^{\mathbf{D n}}$ at ca. 34.2 ppm shows H-D scrambling. 


$$
\begin{aligned}
& \oplus
\end{aligned}
$$

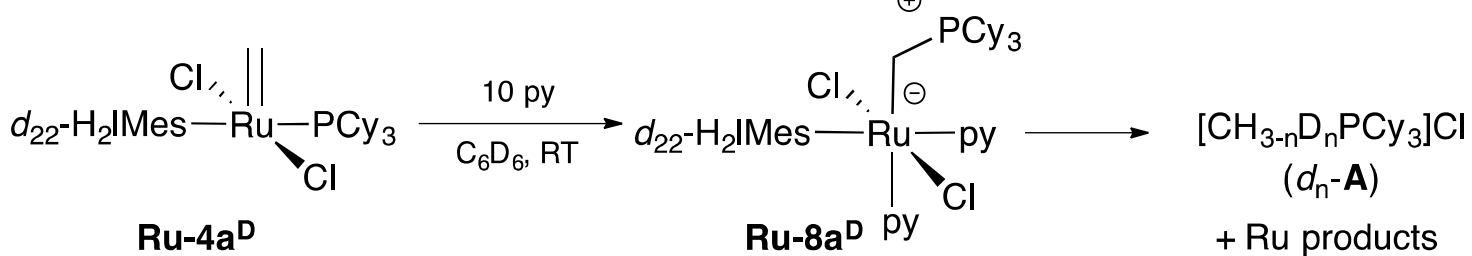

(a)

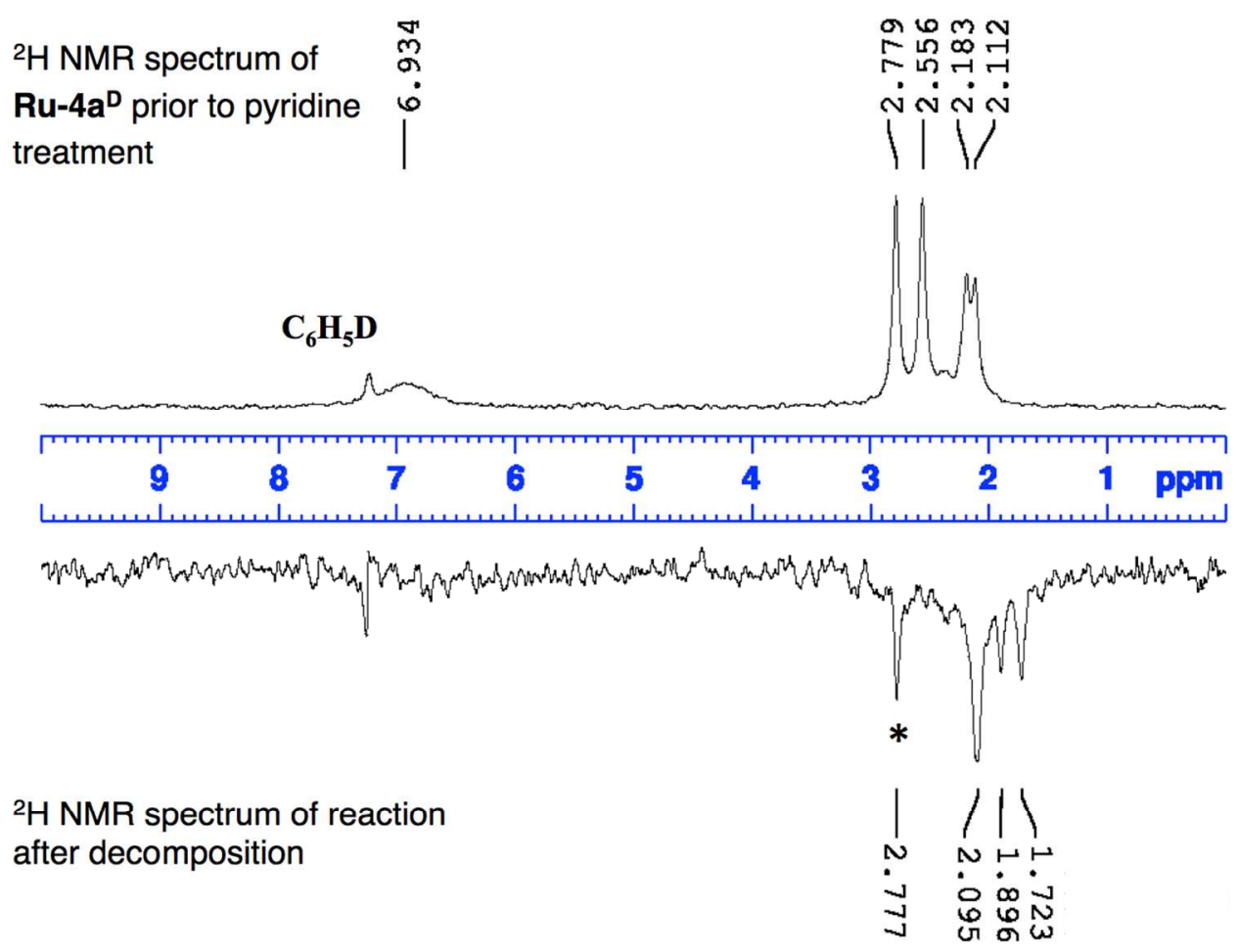

(b)

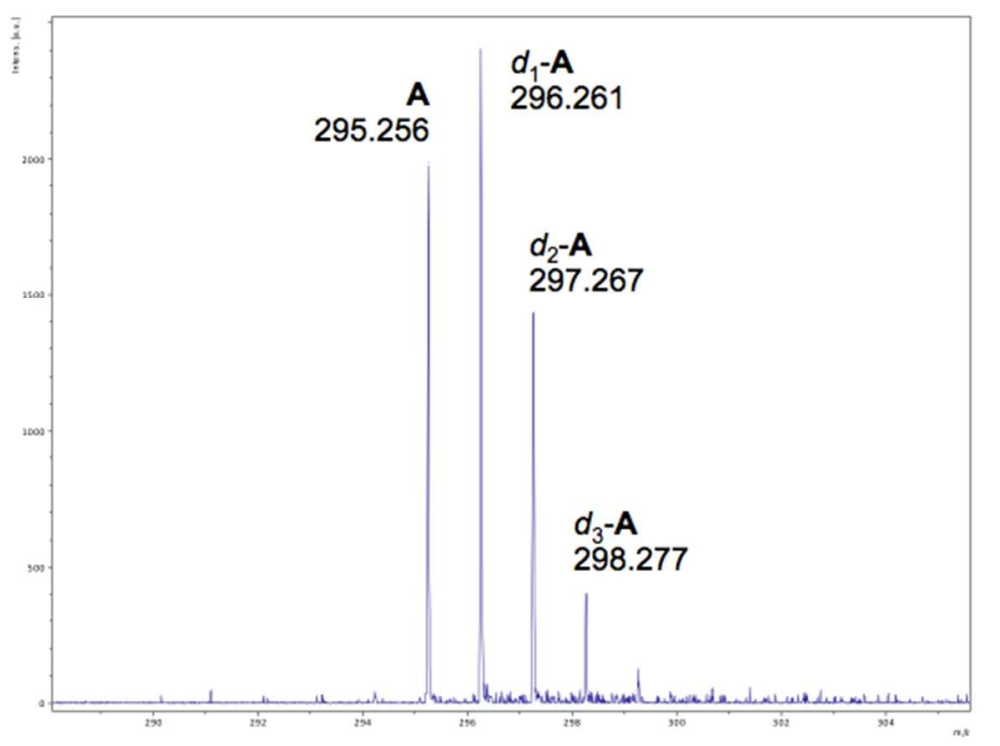

Figure S10. (a) (a) ${ }^{2} \mathrm{H}$ NMR spectra $\left(\mathrm{C}_{6} \mathrm{H}_{6}, 46 \mathrm{MHz}\right)$ of $\mathbf{R u}-\mathbf{4} \mathbf{a}^{\mathbf{D}}$ prior to and (inverted) following decomposition by pyridine. [*] indicates $\left[\mathrm{CH}_{2} \mathrm{DPCy}_{3}\right] \mathrm{Cl}$ singlet. (b) MALDI-TOF mass spectrum (pyrene matrix) ${ }^{2}$ showing isotopomers of $\mathbf{A}$. 
S4. Donor-Accelerated Methylidene Abstraction: Scope with Respect to Catalyst. Representative NMR Spectra and Tabulated Data

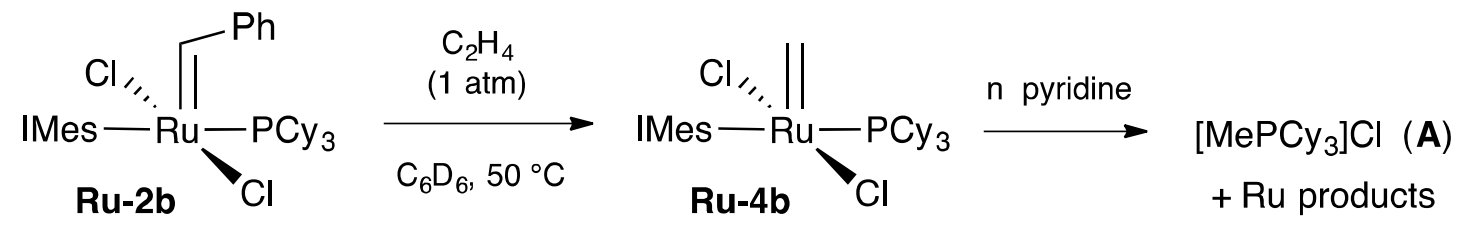

(a) Ru-2b under ethylene; no added pyridine.

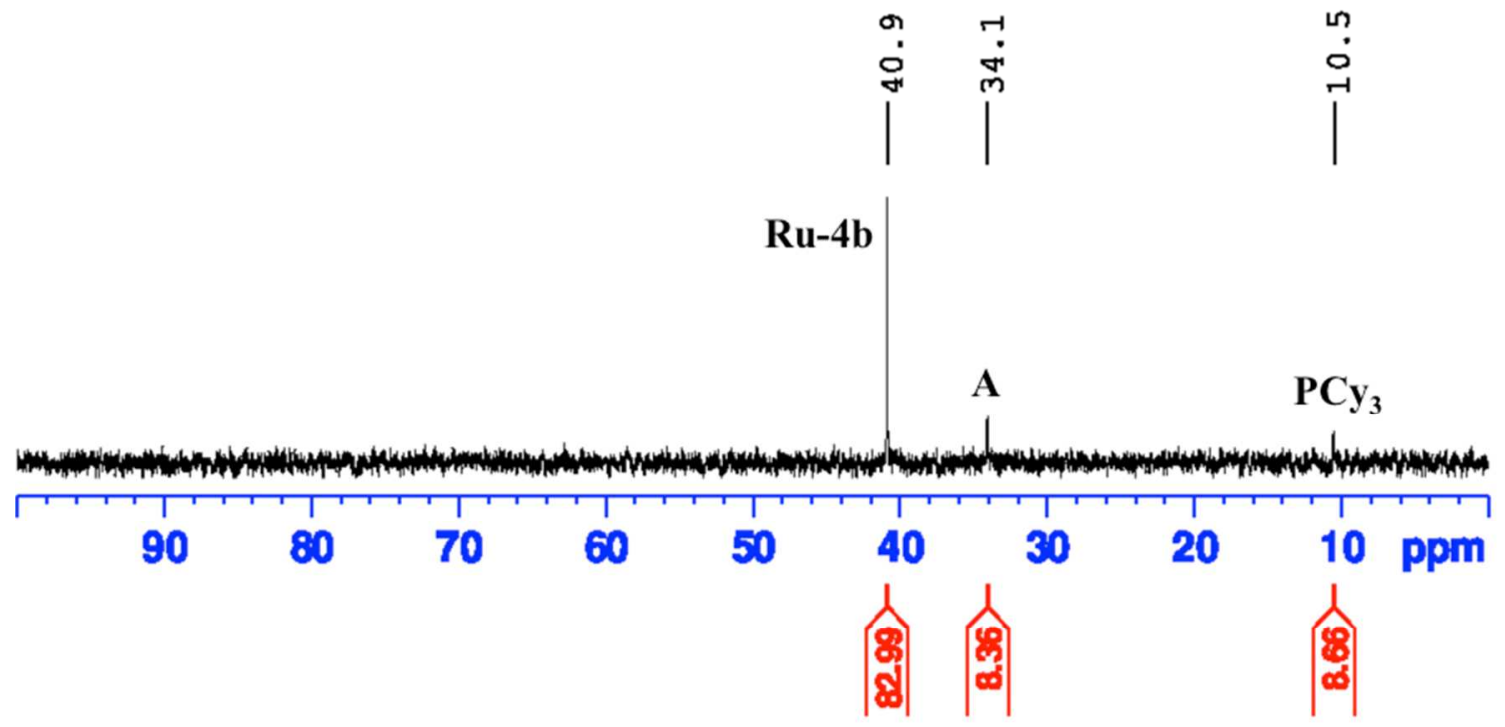

(b) Ru-2b under ethylene;10 equiv added pyridine.

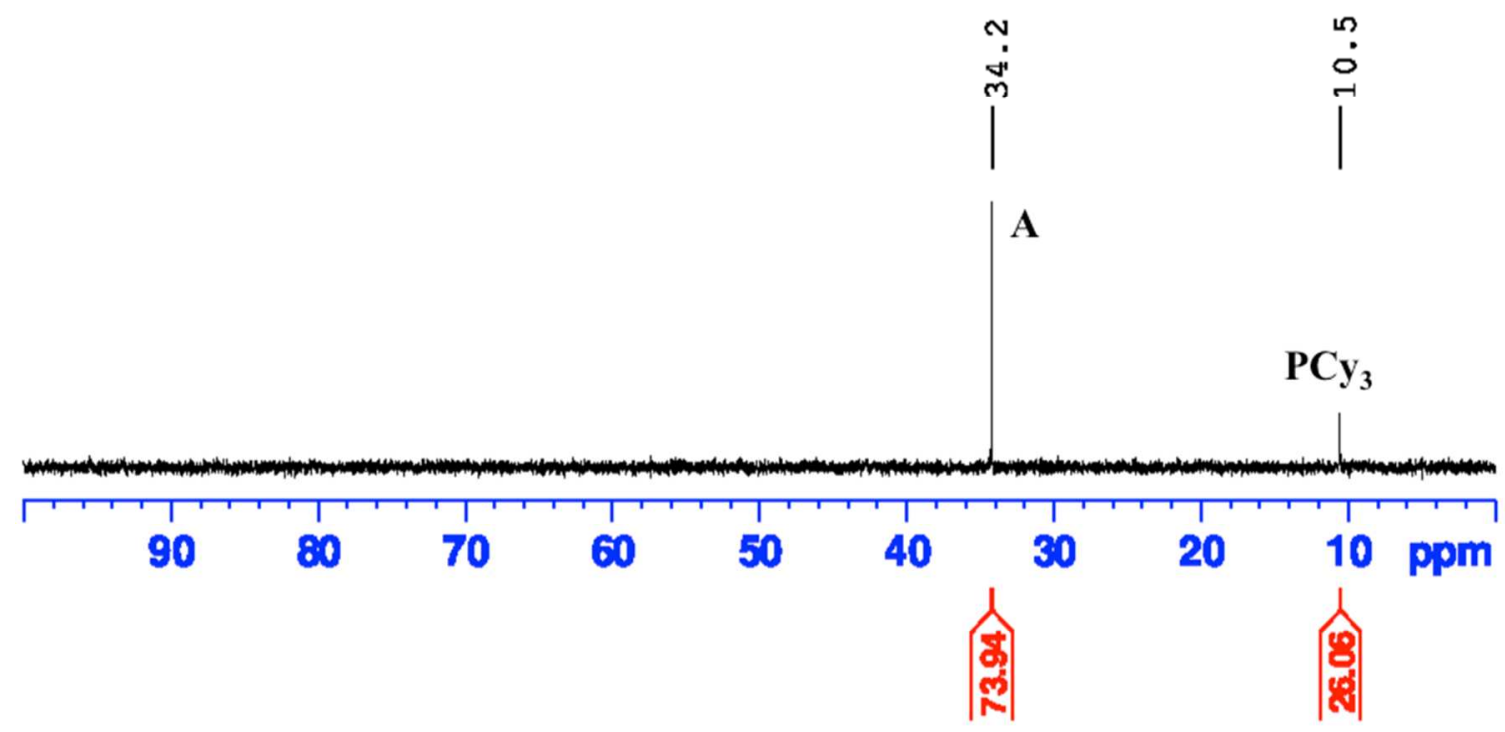

Figure S11. Representative ${ }^{31} \mathrm{P}\left\{{ }^{1} \mathrm{H}\right\}$ NMR spectra $\left(121 \mathrm{MHz}, \mathrm{C}_{6} \mathrm{D}_{6}\right)$ showing the impact of pyridine on decomposition of $\mathbf{R u - 2 b}$ under ethylene. Spectra after $2 \mathrm{~h}$. See Figure 3 in main text. 
Table S1. ${ }^{31} \mathrm{P}$ NMR data demonstrating accelerating effect of pyridine on decomposition of phosphine-stabilized catalysts under ethylene (Figure 3 in text).

\begin{tabular}{|c|c|c|c|c|c|}
\hline \multirow[t]{2}{*}{ System } & \multirow[t]{2}{*}{ Equiv } & \multicolumn{2}{|c|}{$\%[\mathrm{Ru}]=\mathrm{CHR} \quad\left(\delta_{\mathrm{P}}\right)$} & \multirow{2}{*}{$\begin{array}{c}\% \mathbf{A} \\
\left(\delta_{\mathrm{P}} 34.2\right)\end{array}$} & \multirow{2}{*}{$\begin{array}{l}\% \mathrm{PCy}_{3} \\
\left(\delta_{\mathrm{P}} 10.5\right)\end{array}$} \\
\hline & & $\mathrm{R}=\mathrm{Ph}$ & $\mathrm{R}=\mathrm{H}$ & & \\
\hline \multirow[t]{2}{*}{ Ru-2a } & 0 & 0 & $62\left(\delta_{\mathrm{P}} 38.2\right)$ & 38 & 0 \\
\hline & 10 & 0 & 0 & 100 & 0 \\
\hline \multirow[t]{2}{*}{$\mathbf{R u}-\mathbf{2 b}$} & 0 & 0 & $83\left(\delta_{\mathrm{P}} 40.9\right)$ & 8 & 9 \\
\hline & 10 & 0 & 0 & 74 & 26 \\
\hline \multirow[t]{2}{*}{ Ru-2c } & 0 & 0 & $29(38.4)$ & 71 & 0 \\
\hline & 10 & 0 & 0 & 100 & 0 \\
\hline \multirow[t]{2}{*}{ Ru-2d } & 0 & 0 & $85\left(\delta_{\mathrm{P}} 40.4\right)$ & 15 & 0 \\
\hline & 10 & 0 & 0 & 100 & 0 \\
\hline \multirow[t]{2}{*}{ Ru-2f } & 0 & $19\left(\delta_{\mathrm{P}} 26.5\right)$ & $69\left(\delta_{\mathrm{P}} 38.2\right)$ & 12 & 0 \\
\hline & 10 & 0 & 0 & 91 & 9 \\
\hline \multirow[t]{2}{*}{ Ru-2g } & 0 & $86\left(\delta_{\mathrm{P}} 28.1\right)$ & $14\left(\delta_{\mathrm{P}} 40.9\right)$ & 0 & 0 \\
\hline & 10 & 0 & 0 & 82 & 18 \\
\hline \multirow[t]{2}{*}{$\mathbf{R u}-\mathbf{2} \mathbf{e}^{a}$} & 0 & 0 & 0 & 51 & 0 \\
\hline & 10 & 0 & 0 & 81 & 6 \\
\hline
\end{tabular}


S5. Donor-Accelerated Methylidene Abstraction: Scope with Respect to Donor. Representative NMR Spectra

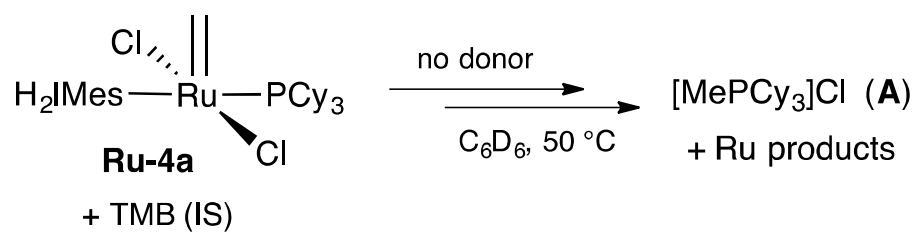

(a) Spectra obtained immediately after dissolving the sample.
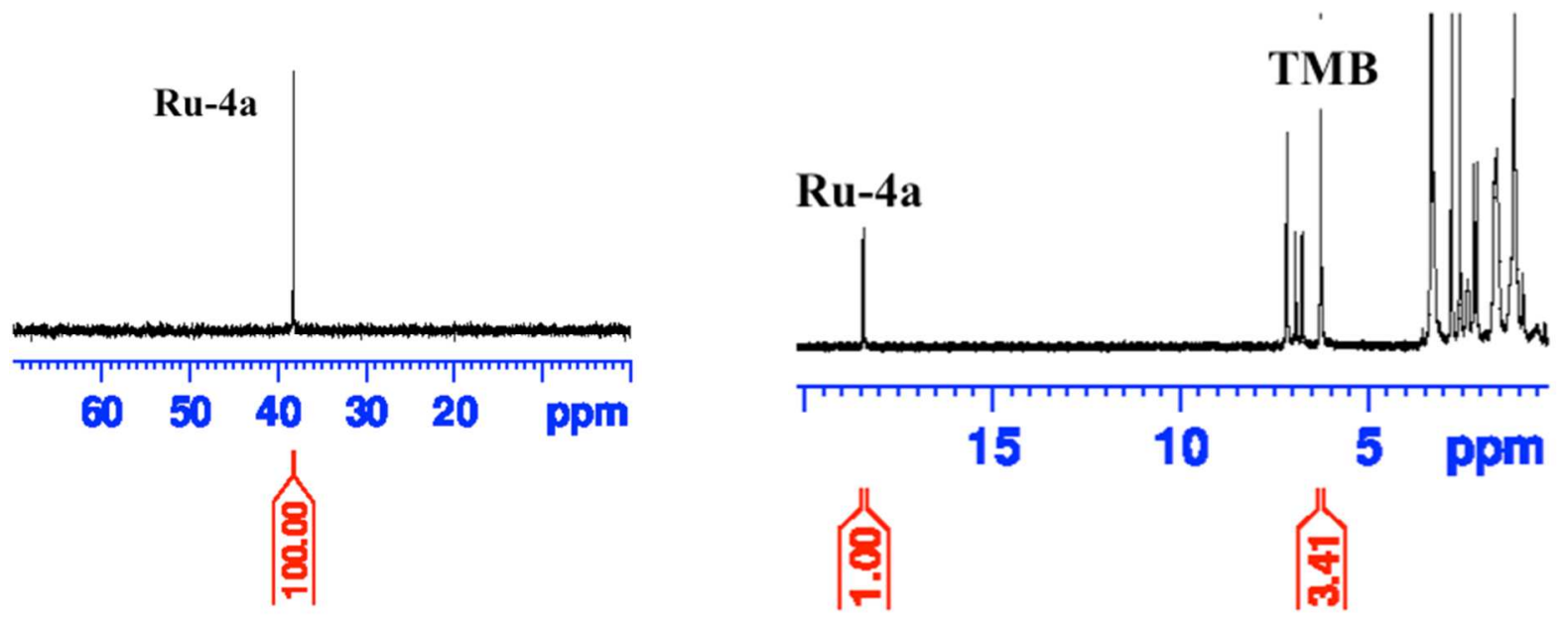

(b) Spectra after $2 \mathrm{~h}$ at $50{ }^{\circ} \mathrm{C}$.
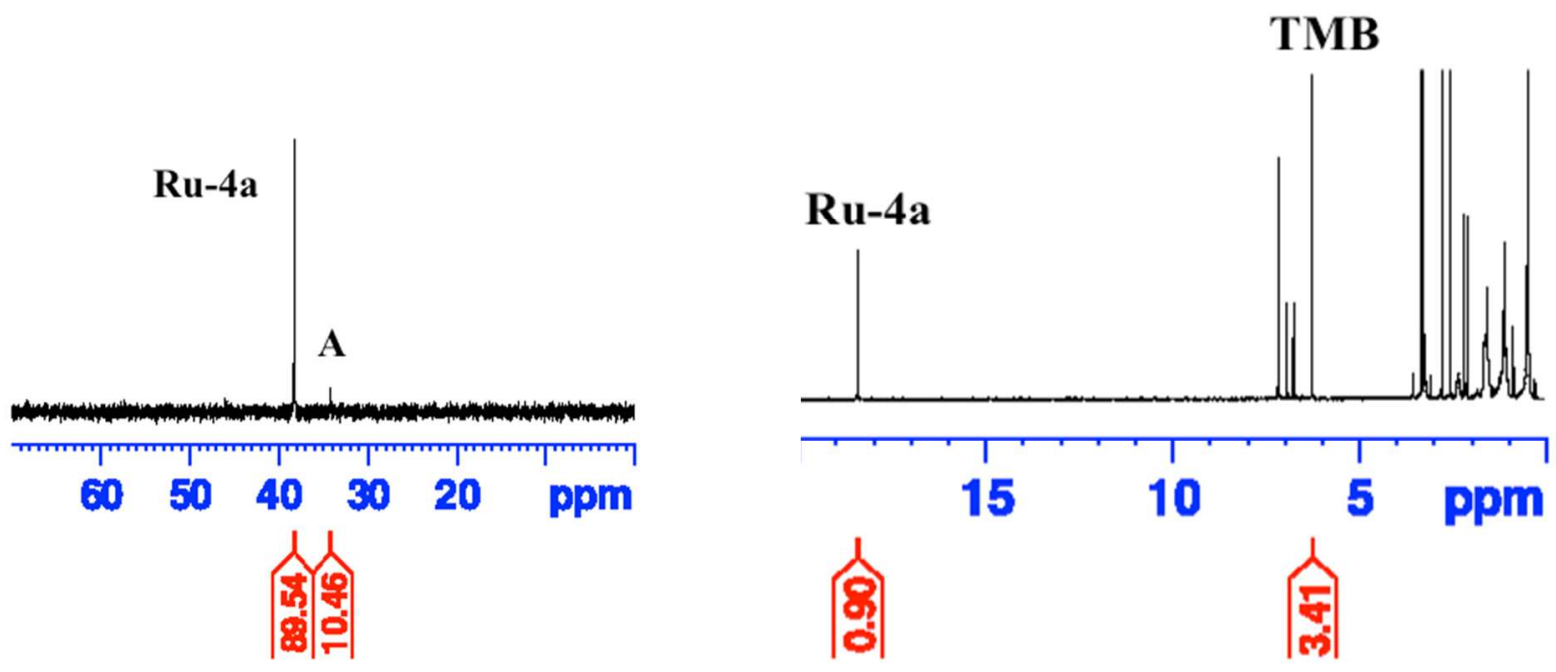

Figure S12. Reaction (top) and representative NMR spectra (left, ${ }^{31} \mathrm{P}\left\{{ }^{1} \mathrm{H}\right\}$; right, ${ }^{1} \mathrm{H}$ ) showing disappearance of methylidene complex $\mathbf{R u}-\mathbf{4 a}$, and formation of minor amounts of $\left[\mathrm{MePCy}_{3}\right] \mathrm{Cl}$ A, in the absence of any added donor. See Table 1 in main text. 


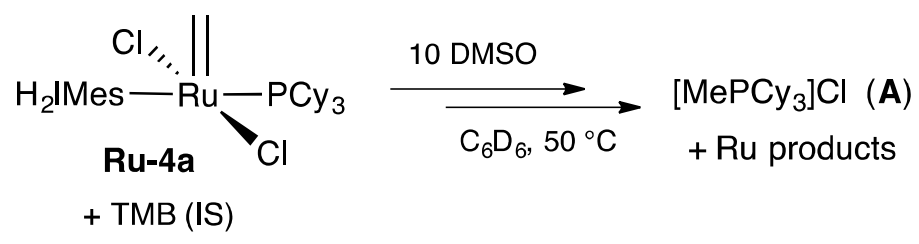

(a) Immediately following sample preparation. Left: ${ }^{31} \mathrm{P}\left\{{ }^{1} \mathrm{H}\right\}$ spectrum; right: ${ }^{1} \mathrm{H}$ NMR spectrum.
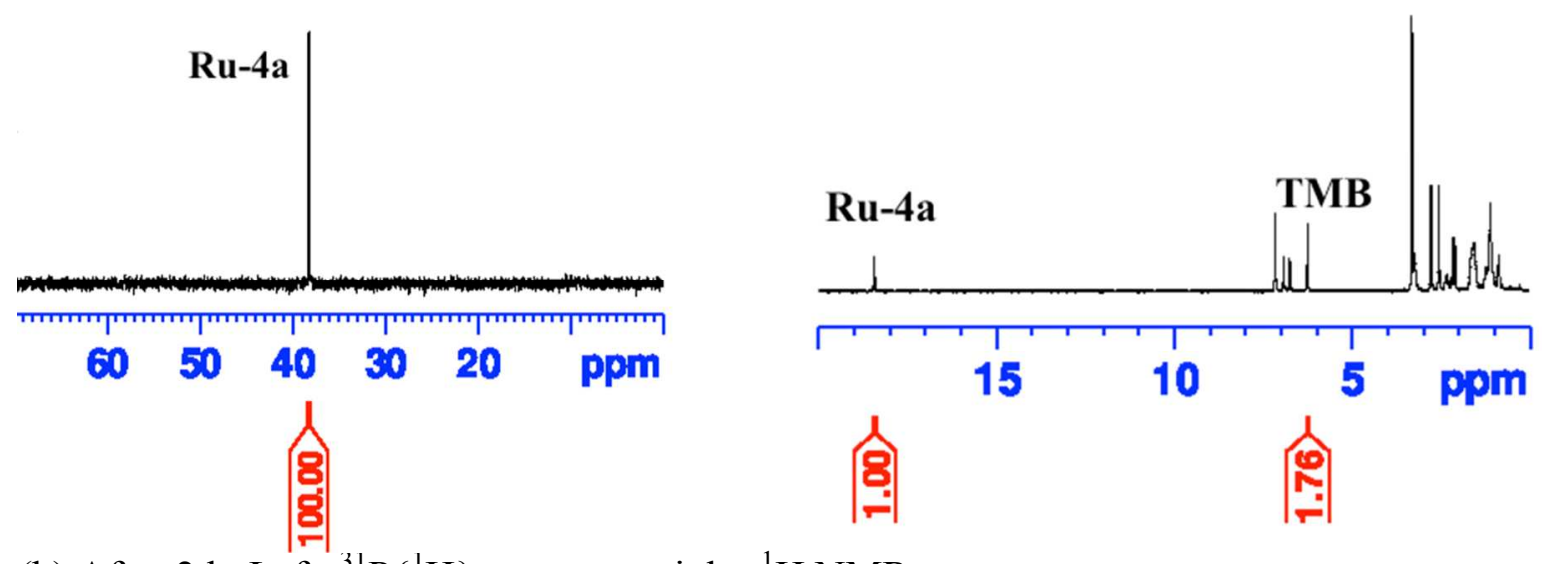

(b) After 2 h. Left: ${ }^{31} \mathrm{P}\left\{{ }^{1} \mathrm{H}\right\}$ spectrum; right: ${ }^{1} \mathrm{H}$ NMR spectrum.
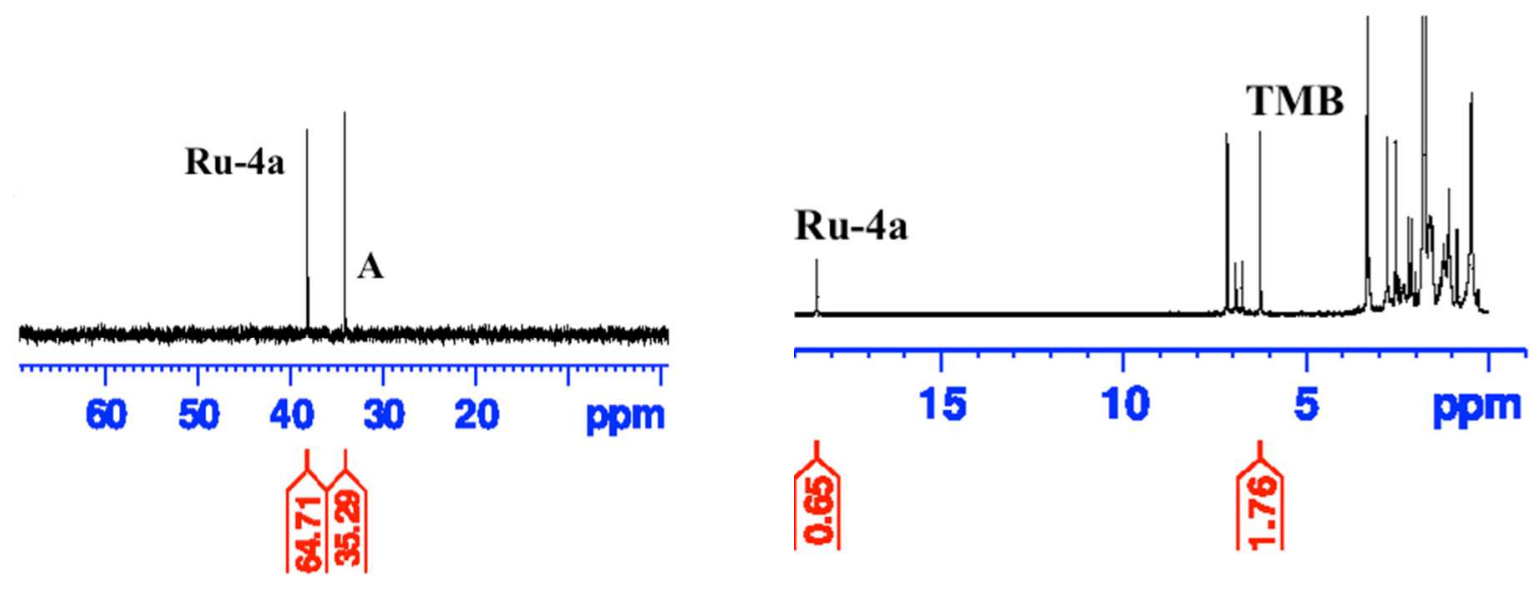

Figure S13. Representative spectra showing loss of $\mathbf{R u}-4 \mathbf{a}$ and formation of $\left[\mathrm{MePCy}_{3}\right] \mathrm{Cl} \mathbf{A}$ on treating with $n$ equiv donor (here DMSO, $n=10$ ) at $50^{\circ} \mathrm{C}$. See Table 1 in main text.

Note: for spectra showing decomposition of ${ }^{13} \mathrm{C}$-labelled $\mathrm{RuCl}_{2}\left(\mathrm{H}_{2} \mathrm{IMes}\right)\left(\mathrm{PCy}_{3}\right)\left(={ }^{13} \mathrm{CH}_{2}\right) * \mathbf{R u}-\mathbf{4 a}$ on treatment with pyridine, see the prior Communication. ${ }^{1}$ 


\section{S6. Kinetics Data for Decomposition of Methylidene Complexes}

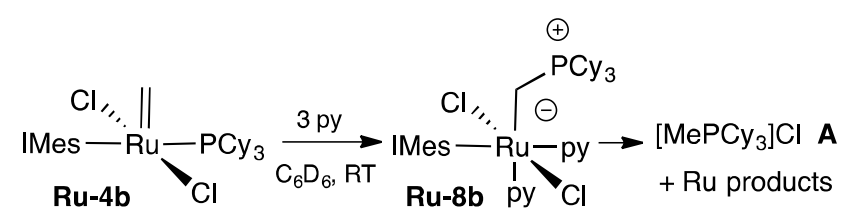

(a)

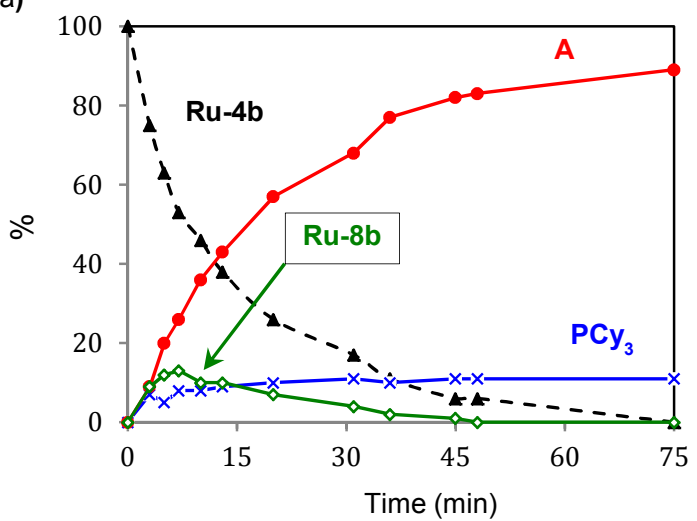

(b)

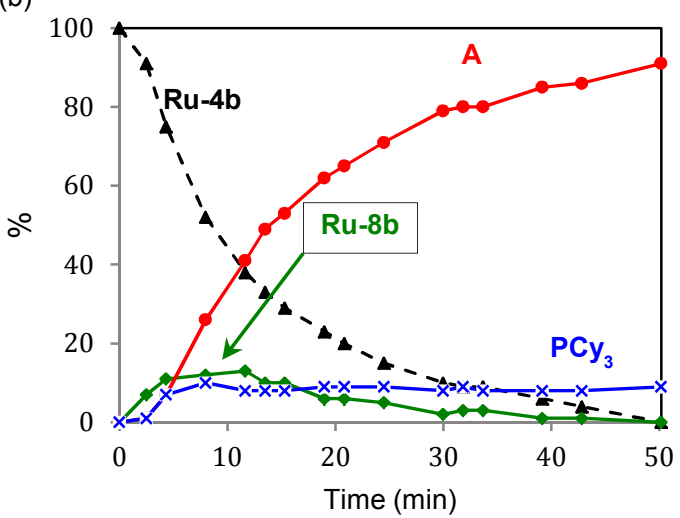

Figure S14. Rate plots for decomposition of Ru-4b by pyridine. (a) Reproduced from Figure 1 in text. (b) Accelerating effect of added $\mathrm{PCy}_{3}$ (10 equiv). Conditions $20 \mathrm{mM}[\mathrm{Ru}], \mathrm{RT}=22^{\circ} \mathrm{C}$.

Table S2. Tabulated ${ }^{31} \mathrm{P}\left\{{ }^{1} \mathrm{H}\right\}$ data $\left(121 \mathrm{MHz}, \mathrm{C}_{6} \mathrm{D}_{6}\right)$ for Figure $\mathrm{S} 14 \mathrm{a}$ (corresponds to Figure 1 in main text). Observation of $\mathbf{R u - 8 b}$ on treatment of $\mathbf{R u - 4 b}$ with 3 equiv pyridine. ${ }^{a}$

\begin{tabular}{ccccc}
\hline time $(\mathrm{min})$ & $\begin{array}{c}\text { \% } \mathbf{R u}-\mathbf{4 b} \\
\left(\delta_{\mathrm{P}} 40.9\right)\end{array}$ & $\begin{array}{c}\% \mathbf{R u - 8 b} \\
\left(\delta_{\mathrm{P}} 61.3\right)\end{array}$ & $\begin{array}{c}\%\left[\mathrm{MePCy}_{3}\right] \mathrm{Cl}, \mathbf{A} \\
\left(\delta_{\mathrm{P}} 34.2\right)\end{array}$ & $\begin{array}{c}\% \text { free } \mathrm{PCy}_{3} \\
\left(\delta_{\mathrm{P}} 10.5\right)\end{array}$ \\
\hline 0 & 100 & 0 & 0 & 0 \\
3 & 75 & 9 & 9 & 7 \\
5 & 63 & 12 & 20 & 8 \\
7 & 53 & 13 & 26 & 8 \\
10 & 46 & 10 & 36 & 9 \\
13 & 38 & 10 & 43 & 10 \\
20 & 26 & 7 & 57 & 10 \\
31 & 17 & 4 & 68 & 10 \\
36 & 11 & 2 & 77 & 11 \\
45 & 6 & 1 & 82 & 11 \\
48 & 6 & 0 & 83 & 11 \\
75 & 0 & 0 & 89 & \\
\hline
\end{tabular}

${ }^{a}$ Values in red correspond to spectrum shown in Figure S7, p. S7. 
Table S3. Tabulated ${ }^{31} \mathrm{P}\left\{{ }^{1} \mathrm{H}\right\}$ data $\left(121 \mathrm{MHz}, \mathrm{C}_{6} \mathrm{D}_{6}\right)$ for Figure S14b, showing accelerating effect of $\left[\mathrm{PCy}_{3}\right]$ on rate of decomposition of $\mathbf{R u}-\mathbf{4 b}$ by pyridine.

\begin{tabular}{ccccc}
\hline time $(\min )$ & $\begin{array}{c}\% \mathbf{R u}-\mathbf{4 b} \\
\left(\delta_{\mathrm{P}} 40.9\right)\end{array}$ & $\begin{array}{c}\% \mathbf{R u - 8 b} \\
\left(\delta_{\mathrm{P}} 61.3\right)\end{array}$ & $\begin{array}{c}\%\left[\mathrm{MePCy}_{3}\right] \mathrm{Cl} \mathrm{A} \\
\left(\delta_{\mathrm{P}} 34.2\right)\end{array}$ & $\begin{array}{c}\% \text { free } \mathrm{PCy}_{3} \\
\left(\delta_{\mathrm{P}} 10.5\right)^{a}\end{array}$ \\
\hline 0 & 100 & 0 & 0 & 0 \\
2 & 91 & 7 & 1 & 1 \\
4 & 75 & 11 & 7 & 10 \\
8 & 52 & 12 & 26 & 8 \\
12 & 38 & 13 & 41 & 8 \\
14 & 33 & 10 & 49 & 8 \\
15 & 29 & 10 & 53 & 9 \\
19 & 23 & 6 & 62 & 9 \\
21 & 20 & 6 & 65 & 9 \\
24 & 15 & 5 & 71 & 8 \\
30 & 10 & 2 & 79 & 8 \\
32 & 9 & 3 & 80 & 8 \\
34 & 9 & 3 & 80 & 9 \\
39 & 6 & 1 & 85 & 96 \\
43 & 4 & 1 & 91 & 9 \\
50 & 0 & 0 & 6 & 9 \\
\hline
\end{tabular}

${ }^{a}$ These values were calculated after accounting for the 10 equiv added $\mathrm{PCy}_{3}$ by subtracting the initial $\mathrm{PCy}_{3}$ integration at $\mathrm{t}=0 \mathrm{~min}$. 
Table S4. Kinetics data showing disappearance of methylidene species on reaction with DMSO at $25{ }^{\circ} \mathrm{C}$. See Figure $4 \mathrm{~b}$ in main text.

\begin{tabular}{|c|c|c|c|}
\hline Equiv. DMSO & time (h) & $\% \mathbf{R u}-\mathbf{6}^{a}$ & $\% \mathbf{R u}-\mathbf{4 \mathbf { a } ^ { a }}$ \\
\hline \multirow{6}{*}{10} & 0 & 100 & 100 \\
\hline & 1 & 81 & 99 \\
\hline & 2 & 72 & 97 \\
\hline & 4 & 53 & 93 \\
\hline & 6 & 43 & 90 \\
\hline & 27 & 9 & 64 \\
\hline \multirow[t]{6}{*}{33} & 0 & 100 & 100 \\
\hline & 1 & 53 & 92 \\
\hline & 2 & 33 & 91 \\
\hline & 4 & 17 & 87 \\
\hline & 6 & 9 & 81 \\
\hline & 24 & 0 & 57 \\
\hline \multirow[t]{6}{*}{55} & 0 & 100 & 100 \\
\hline & 1 & 44 & 78 \\
\hline & 2 & 24 & 76 \\
\hline & 4 & 2 & 64 \\
\hline & 6 & 0 & 60 \\
\hline & 27 & 0 & 21 \\
\hline \multirow[t]{6}{*}{78} & 0 & 100 & 100 \\
\hline & 1 & 49 & 77 \\
\hline & 2 & 14 & 73 \\
\hline & 4 & 5 & 58 \\
\hline & 6 & 0 & 48 \\
\hline & 27 & 0 & 13 \\
\hline \multirow[t]{6}{*}{100} & 0 & 100 & 100 \\
\hline & 1 & 30 & 77 \\
\hline & 2 & 5 & 71 \\
\hline & 4 & 0 & 56 \\
\hline & 6 & 0 & 36 \\
\hline & 27 & 0 & 6 \\
\hline
\end{tabular}

${ }^{a}$ Integrated ${ }^{1} \mathrm{H}$ NMR analysis vs. TMB. 
Table S5. Half-lives $(\mathrm{min})$ and rate constants $\left(\mathrm{min}^{-1}\right)$ for kinetics data in Table S4, showing decomposition of Ru-6 and Ru-4a by DMSO.

\begin{tabular}{lll}
\hline & Ru-6 & Ru-4a \\
equiv DMSO & $t_{1 / 2}\left(k_{\mathrm{obs}}\right)$ & $t_{1 / 2}\left(k_{\mathrm{obs}}\right)$ \\
\hline 10 & $250(0.0016)$ & $1,800(0.0003)$ \\
33 & $70(0.0062)$ & $1,700(0.0004)$ \\
55 & $50(0.011)$ & $420(0.0009)$ \\
78 & $40(0.014)$ & $330(0.0012)$ \\
100 & $30(0.017)$ & $270(0.0015)$ \\
\hline
\end{tabular}

\section{S7. NMR Spectra Relating to Points Discussed in Text}

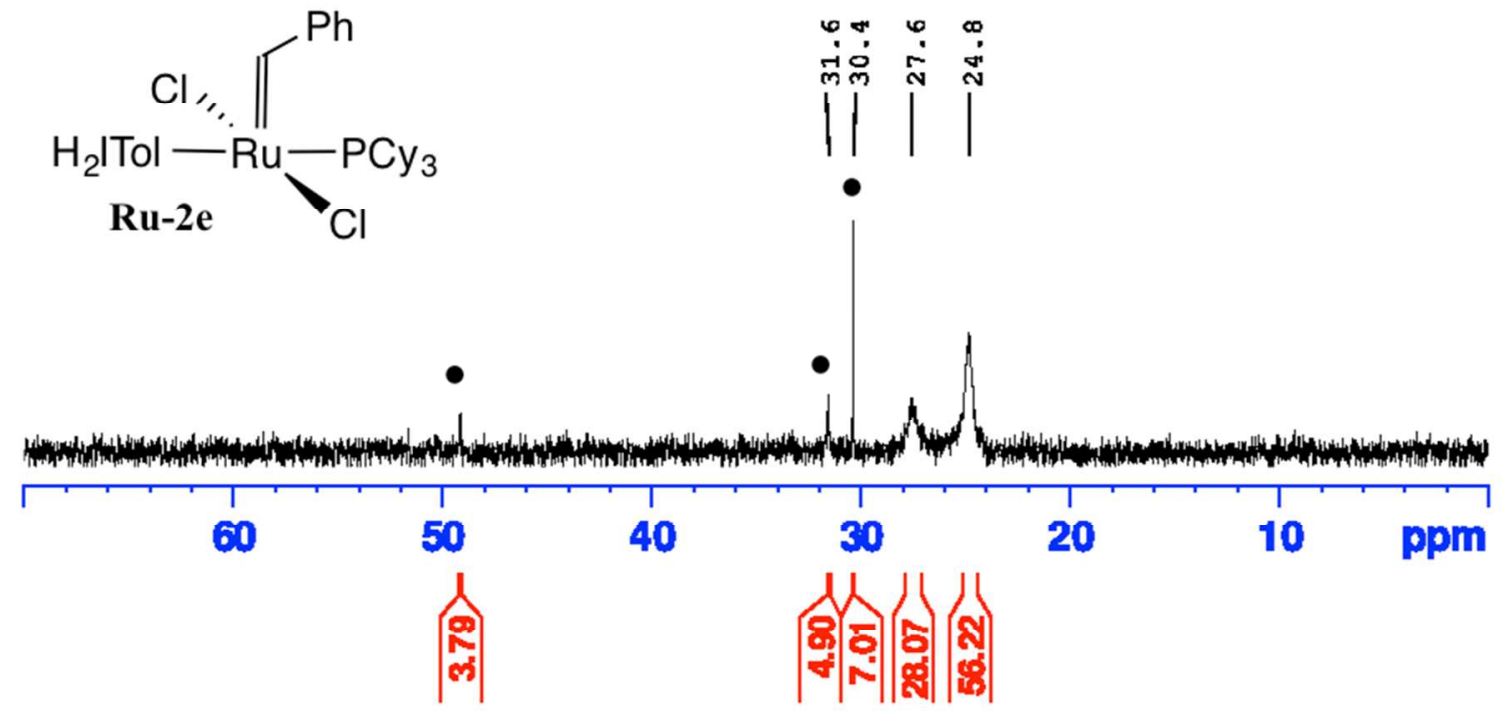

Figure S15. ${ }^{31} \mathrm{P}\left\{{ }^{1} \mathrm{H}\right\}$ NMR spectrum $\left(121 \mathrm{MHz}, \mathrm{C}_{6} \mathrm{D}_{6}\right)$ of commercial $\mathrm{H}_{2}$ ITol complex Ru-2e, showing the two signals reported, ${ }^{3}$ and unidentified impurities labelled $(\bullet)$. See Figure 3 and accompanying discussion in main text. 


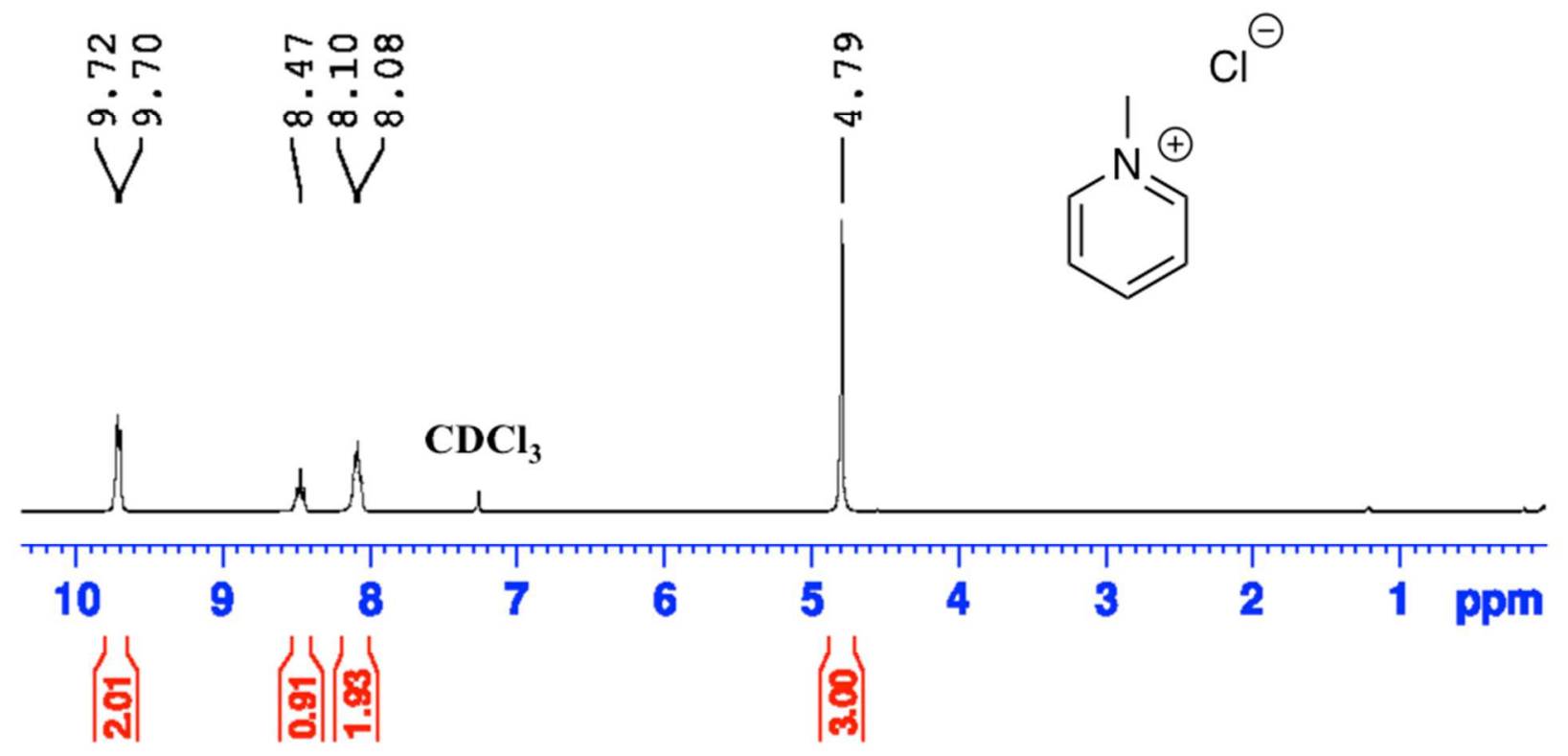

Figure S16. ${ }^{1} \mathrm{H}$ NMR $\left(300 \mathrm{MHz}, \mathrm{CDCl}_{3}\right)$ spectrum of commercially available $\left[\mathrm{MeNC}_{5} \mathrm{H}_{5}\right] \mathrm{Cl}$. See Ref. 31 in main text, and associated discussion.

\section{S8. Representative GC Data}

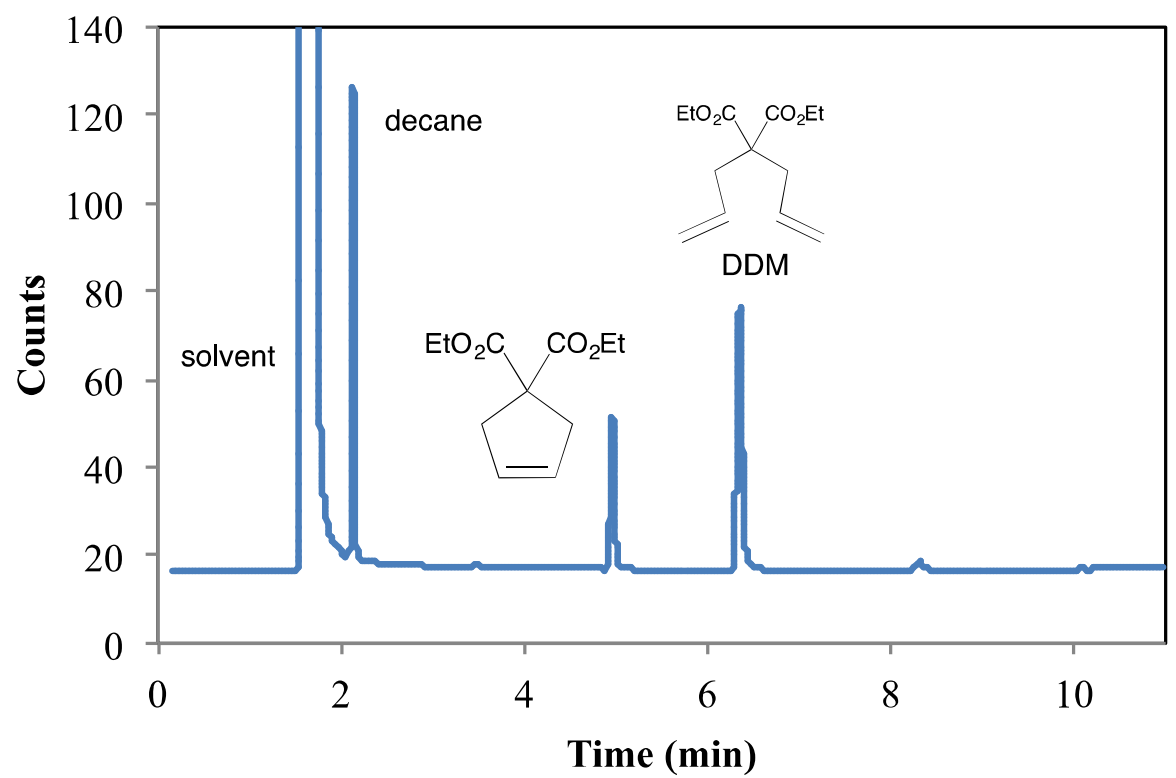

Figure S17. GC-FID trace for RCM of DDM by Ru-2a in the presence of $90 \mu \mathrm{L}$ water at $2 \mathrm{~h}$. 
S9. NMR Spectra for Experiments with Ethylidene Complexes

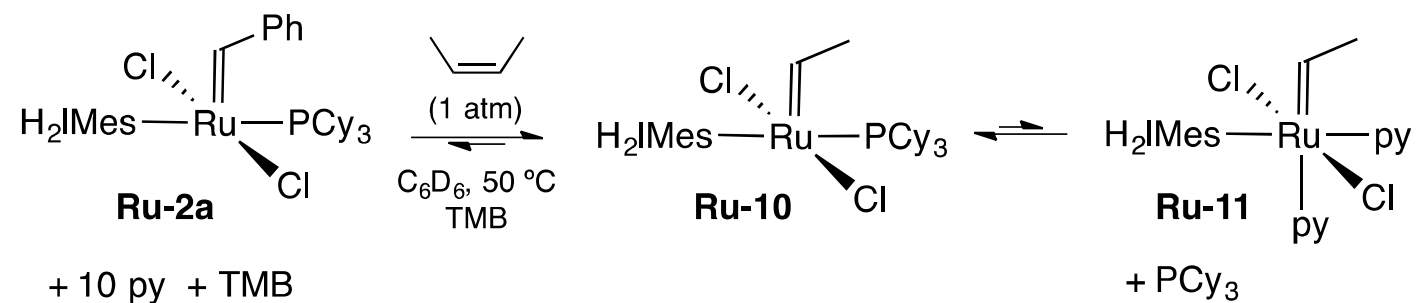

(a) ${ }^{1} \mathrm{H}$ NMR spectrum showing in situ-generated ethylidene species $\mathbf{R u - 1 0}$ and $\mathbf{R u - 1 1}$.

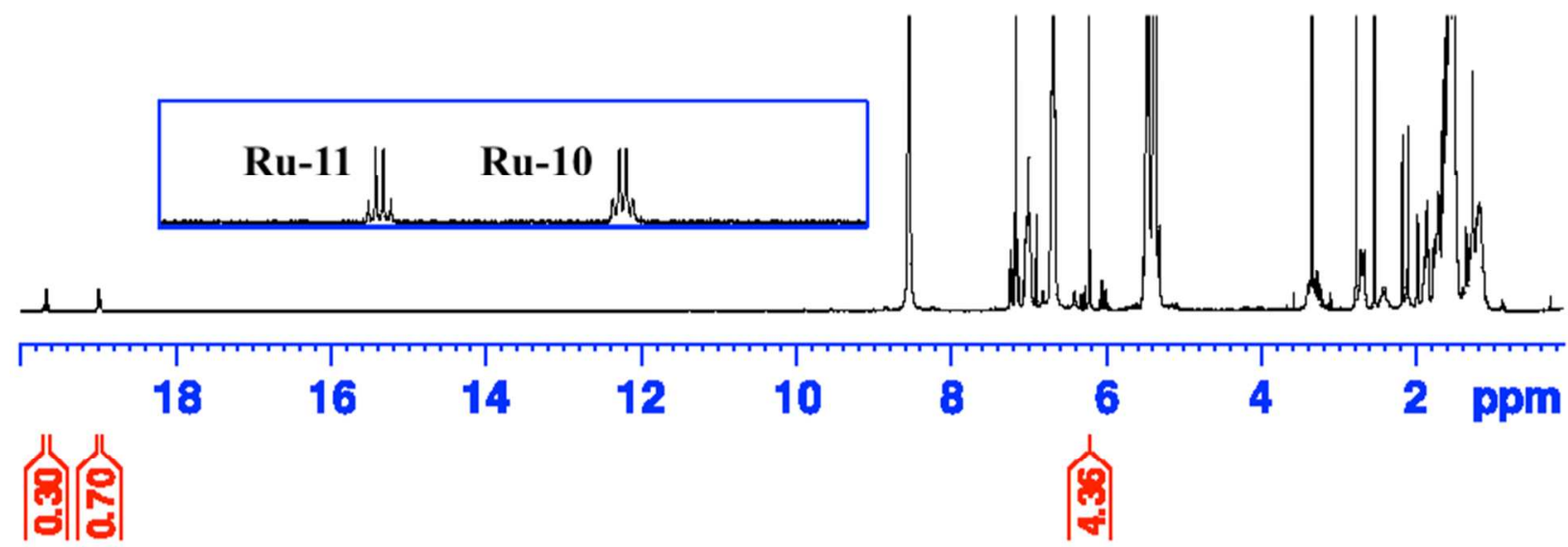

(b) ${ }^{31} \mathrm{P}\left\{{ }^{1} \mathrm{H}\right\}$ NMR spectrum showing $\mathbf{R u - 1 0}$ and free $\mathrm{PCy}_{3}$.

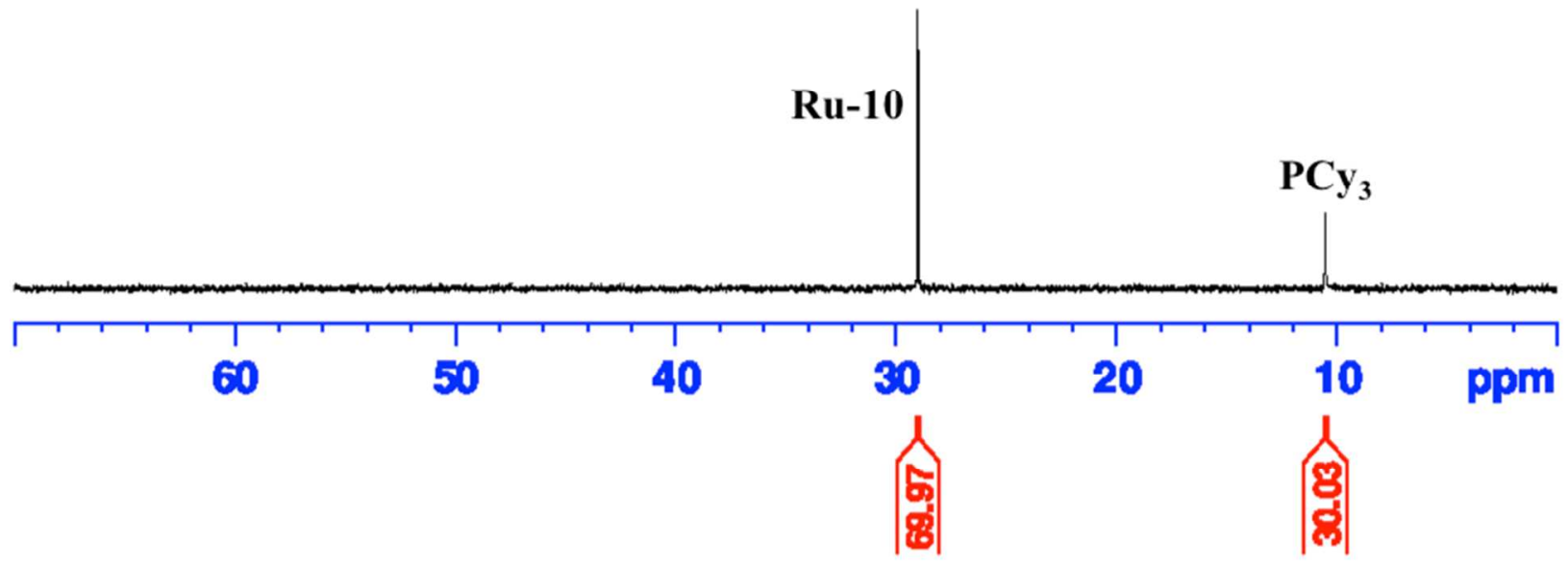

Figure S18. Reaction (top) and NMR spectra $\left({ }^{1} \mathrm{H}: 300 \mathrm{MHz} ;{ }^{31} \mathrm{P}: 121 \mathrm{MHz} ; \mathrm{C}_{6} \mathrm{D}_{6}\right)$ showing ethylidene species generated from Ru-2a after $1 \mathrm{~h}$ at $50^{\circ} \mathrm{C}$. See Scheme 5 in text. 


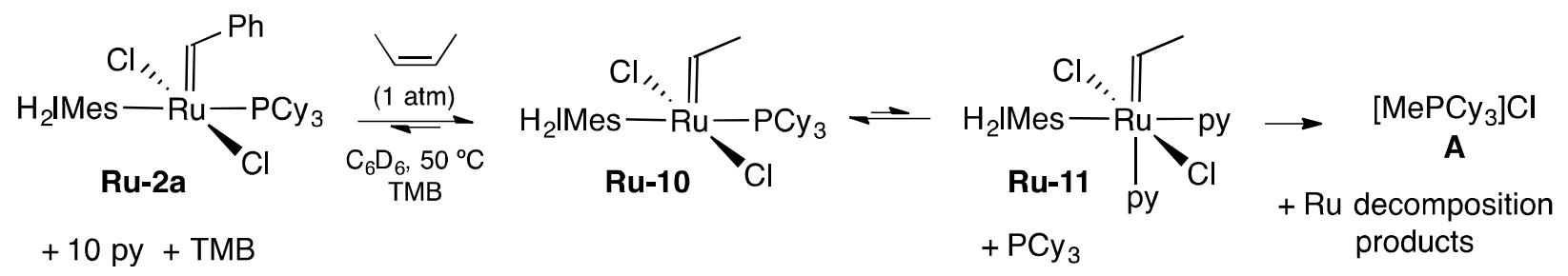

(a) ${ }^{1} \mathrm{H}$ NMR spectrum showing complete loss of $[\mathrm{Ru}]=$ CHR species. $(\bullet)$ indicates 1 -butene.

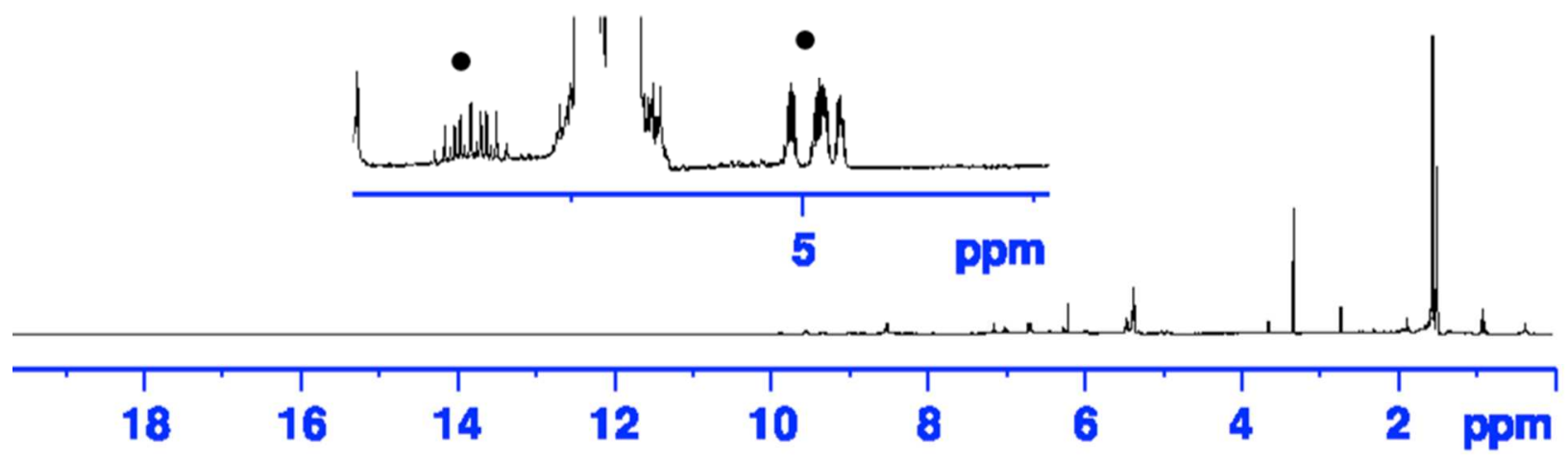

(b) ${ }^{31} \mathrm{P}\left\{{ }^{1} \mathrm{H}\right\}$ NMR spectrum showing only $\left[\mathrm{MePCy}_{3}\right] \mathrm{Cl} \mathbf{A}$ in the phosphonium salt region. Assignment confirmed by MALDI-TOF MS. The signal at $47.2 \mathrm{ppm}$ is unassigned.

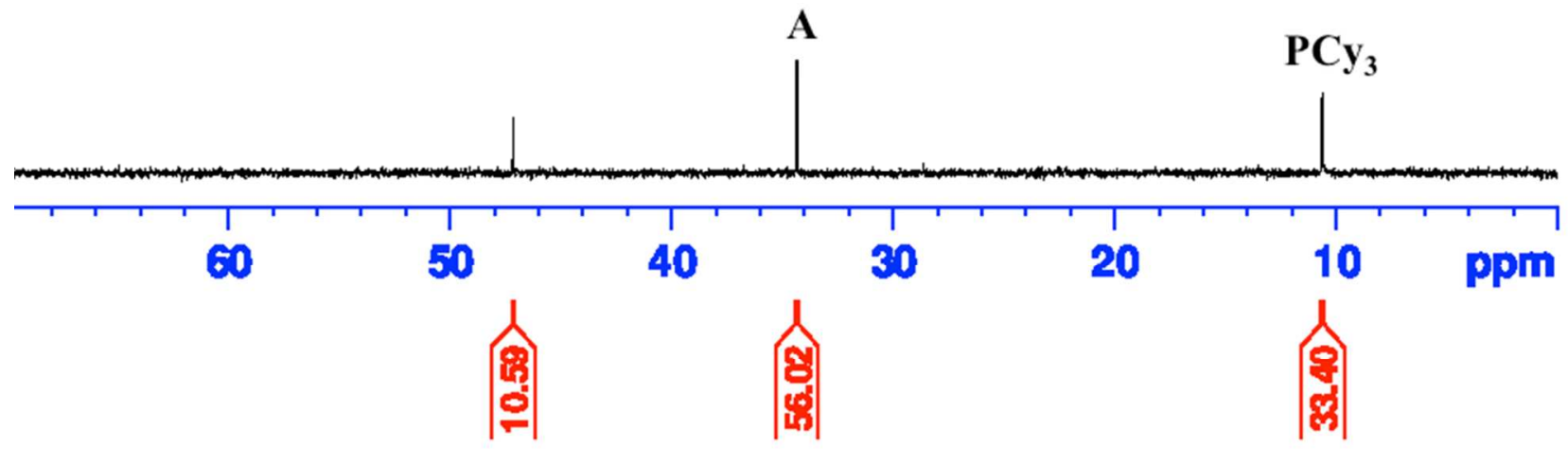

Figure S19. Reaction (top) and NMR spectra showing decomposition products formed from ethylidene species after $48 \mathrm{~h}$ at $50{ }^{\circ} \mathrm{C}$. NMR spectra in $\mathrm{C}_{6} \mathrm{D}_{6} ;{ }^{1} \mathrm{H}$ NMR: $300 \mathrm{MHz} ;{ }^{31} \mathrm{P}: 300 \mathrm{MHz}$. 

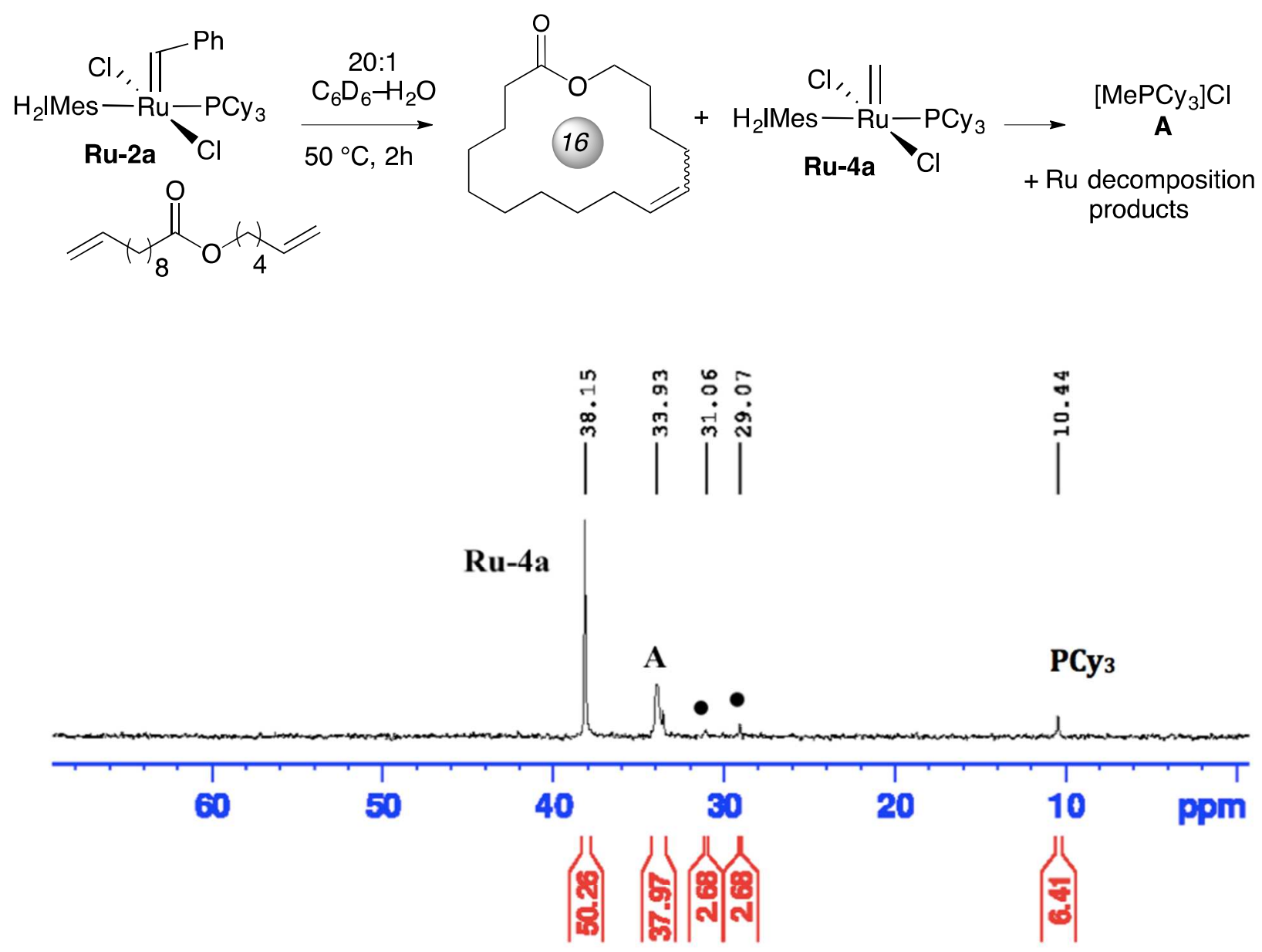

Figure S20. Reaction (top) and ${ }^{31} \mathrm{P}\left\{{ }^{1} \mathrm{H}\right\}$ NMR spectrum (121 MHz, $\mathrm{C}_{6} \mathrm{D}_{6}$ ) showing ca. 40\% decomposition of Ru-2a to $\mathbf{A}$ (based on total integration) during RCM macrocyclization. Spectrum measured after $2 \mathrm{~h}$ at $50^{\circ} \mathrm{C}$. Unidentified decomposition indicated by $(\bullet)$. See Scheme 5 in main text, and accompanying discussion.

\section{S11. References}

(1) Lummiss, J. A. M.; McClennan, W. L.; McDonald, R.; Fogg, D. E. Organometallics 2014, $33,6738-6741$.

(2) Bailey, G. A.; Fogg, D. E. ACS Catal. 2016, 6, 4962-4971.

(3) Stewart, I. C.; Ung, T.; Pletnev, A. A.; Berlin, J. M.; Grubbs, R. H.; Schrodi, Y. Org. Lett. 2007, 9, 1589-1592. 